\title{
Steady flow regimes and mixing performance in arrow-shaped micro-mixers
}

\author{
A. Mariotti, C. Galletti, E. Brunazzi and M.V. Salvetti \\ Dipartimento di Ingegneria Civile e Industriale, \\ University of Pisa, 56122 Pisa, Italy
}

\begin{abstract}
The different flow regimes occurring for increasing Reynolds number $(R e)$ in a arrow-shaped micro-mixer operated for liquid mixing are investigated by the synergic use of experimental flow visualizations and direct numerical simulations. The tilting angle of the arrow-mixer inlet channels with respect to those of the T-shaped mixer is $\alpha=20^{\circ}$. Consistently with previous studies in the literature, it is found that the onset of the engulfment regime, and consequently the sharp increase of the degree of mixing between the two flow streams, occurs at a lower Reynolds number than for a T-mixer operating in the same conditions. However, in contrast to what observed for T-mixers, the degree of mixing does not increase monotonically with $R e$. In fact, at approximately $R e=150$, based on the inlet bulk velocity and the hydraulic diameter of the mixing channel, there is a drop of the mixing degree. This is due to a change in the topology of the vorticity field, and, in particular to the presence of a unique vortical structure in the mixing channel instead of two co-rotating ones typical of the engulfment regime. By further increasing the Reynolds number, the so originated vortical structure in the mixing channel grows in size, and eventually starts to oscillate, leading again to an increase of the degree of mixing, starting from $R e \simeq 170$. Additional simulations have been carried in order to investigate the sensitivity to the tilting angle of the inlet channels, namely for $\alpha=10^{\circ}, 15^{\circ}, 25^{\circ}$. As expected, it is found that the value of $R e$ at which the engulfment regime occurs decreases with increasing $\alpha$. Then, for $\alpha=10^{\circ}$ and $15^{\circ}$, the degree of mixing monotonically increases with the Reynolds numbers and the flow topology in the whole engulfment regime is practically the same as for T-mixers. Conversely, the configuration with $\alpha=25^{\circ}$ shows a behavior of mixing and of flow features with increasing Reynolds number very similar to those observed for $\alpha=20^{\circ}$.
\end{abstract}




\section{INTRODUCTION}

Liquid mixing is one of the key operations in engineering as fast and homogeneous mixing of reactants is a critical step for reactions, dissolutions, precipitations and separations. Micro-mixers, constituted by sub-millimeter channels, have been proposed in the last decade as they allow continuous operation with an unprecedented control over mixing and residence time. One of the main advantages is the enhanced heat transfer capacity due to the high surface to volume ratio. This feature for instance enables safe operation with highly exothermic reactions, thus expanding the number of feasible reactions and conditions that can be run [23] [28]. Moreover, continuous flow reduces accumulation of reactive or toxic intermediates. Hence, microfluidic devices are thought to provide significant economic, environmental and safety benefits for many applications in the fine chemistry and pharmaceutical processes, that have been proposed in addition to more traditional lab-on-chip applications [22]. Since the characteristic dimensions are small, the flow is laminar so mixing should be improved by using special enhancing methods that can be either active or passive [15] [16]. The formers use an extra energy input, such as ultrasound, electric field, pressure pulse, etc. Passive methods, instead, promote mixing by exploiting special designs of the micro-mixer geometry aimed at breaking the flow symmetries; hence they do not need any external energy input.

Among passive micro-mixers, the $\mathrm{T}$ geometry, in which the operating fluid enters the two aligned side channels and then is convected in the mixing channel, having its axis perpendicular to that of the inlet ones, is the most widely used because of its simplicity. For instance, it is often found as junction element in more complex micro-mixer networks. This micro-mixer configuration is also suited to carry out fundamental studies. Indeed, the flow behavior and the mixing efficiency of T-mixers have been largely investigated in the literature. In particular, different flow regimes have been identified as a function of the characteristic flow Reynolds number, Re, usually based on the inlet bulk velocity and on a characteristic length of the mixing channel, as e.g. its hydraulic diameter. At very low $R e$, the two fluid streams flow side by side through the mixing channel, resulting in a completely segregated pattern (stratified regime). Increasing $R e$, in the vortex regime, a double pair of counter-rotating vortical structures are originated in the region of confluence of the two streams and they form four legs of equal strength in the mixing channel. Since the flow in the 
vortical regime preserves the geometrical reflectional symmetries, mixing still occurs only through diffusion and, hence, it remains very low. Further increasing Re, the onset of the so-called engulfment regime takes place. In this regime, a tilting of the vortical structures at the top of the mixer is observed, which leads to a symmetry breaking. Consequently, the two legs of each vortex entering the mixing channel are not equal in terms of intensity and, moving towards the end of the mixing channel, only two co-rotating vortical structures survive. A sudden increase of the degree of mixing is associated with the onset of the engulfment regime, so that many efforts in literature have been devoted at predicting the critical Re at which it occurs and at understanding its dependence on geometric parameters [1], [19], [21], [27], flow conditions [11], [4], [13] and fluid properties [7] [19] [25] [18].

As for the geometrical parameters, the effects of varying the aspect ratio of the channel have been investigated in [1], [19], [21] and [27], showing that this parameter may have a significant impact on the engulfment onset. Other modifications of the T-mixer geometry, aimed at facilitating the onset of the engulfment regime, and, in general, at enhancing mixing, have also been considered in the recent literature [20], [29], [2], [26], [14]. In particular, it was found in [26] that, if the axis of the inlet channels is inclined downward with respect to the mixing channel, in order to obtain a arrow-shaped micro-mixer, the onset of the engulfment regime, and, thus, efficient mixing performance, can be obtained at Reynolds numbers significantly lower than for T-mixers. It was also shown in [26] that Y-shaped mixers are conversely characterized by the opposite trend, i.e. the onset of the engulfment regime occurs at larger Reynolds numbers than for T-mixers. The stability analysis in [26] showed that the differences observed in the flow field for varying the angle between the axis of the inlet channel and the one of the mixing channel, although confined in a small zone, are indeed located inside the core of the instability, i.e. the space region where the instability leading to the engulfment onset originates. This explains the significant effect of even small variations of this angle on the critical Reynolds number value. The simulations in [29] confirmed that the Reynolds number at which complete mixing is achieved in a arrow-shaped mixer is lower than for a T-mixer. However, Galletti et al. [9] observed through numerical simulations a drop in the degree of mixing when the Reynolds number is increased after the onset of the engulfment regime; this decrease in mixing seems to be associated with a change in the topology of the flow and, in particular, of the vorticity field.

The present work is motivated by the results of [26]. Since most of the previously men- 
tioned applications take advantage by a large mixing occurring at low Reynolds numbers, we focus on arrow-shaped mixers, and, in particular, on the largest value of the tilting angle of the mixer inlet channels considered in [26], $\alpha=20^{\circ}$. The investigation in [26] is limited to Reynolds numbers slightly larger than that at which the engulfment regime takes place. Therefore, we carry out herein a systematic and detailed analysis of the flow behavior and mixing performance of this arrow-shaped micro-mixer with increasing Reynolds number up to the onset of unsteady regimes. Direct numerical simulations are used together with experimental flow visualizations. The main goals are (i) to provide a full characterization of the flow and mixing behavior starting from the vortex regime up to Reynolds number values at which the flow becomes unsteady; (ii) to explain the observed behavior in terms of flow topology and physics; (iii) to provide an experimental support to the characterization of flow regimes obtained in numerical simulations, similarly to what done in $[17,24]$ for T-mixers.

\section{EXPERIMENTAL SET UP}

The experimental model is made of polymethylmethacrylate (PMMA) and is shown in Figure 1a. A sketch of the arrow-mixer geometry is reported in Figure 1b, together with the adopted frame of reference. The inlet channels have a square cross-section, with $W_{i}=H=1$ $\mathrm{mm}$, while the mixing channel presents a $2: 1$ aspect ratio, i.e. $W_{o}=2 H=2 \mathrm{~mm}$, so that its hydraulic diameter is $d=4 H / 3$. The length of the inlet channels, $L_{i}=40 \mathrm{~mm} \approx 30 d$ is enough to allow a fully developed flow at the confluence while the length of the mixing channel is $L_{o}=60 \mathrm{~mm} \approx 45 \mathrm{~d}$. The angle between the axis of the inlet channels and the $x$ axis (see Figure $1 \mathrm{~b}$ ) is $\alpha=20^{\circ}$.

The mixer inlets are fed with deionized water streams with equal flow rates, corresponding to a bulk velocity of $U$, by using a KD Scientific syringe pump. The bulk velocity in the mixing channel is therefore also equal to $U$. In order to differentiate the two streams and visualize the mixing process, one inlet stream is colored with a food colorant (E124), dissolved with concentration of $1.9 \cdot 10^{-3} \mathrm{M}$. The mixing of the streams inside the arrowmixer was observed with an upright microscope with a magnifying lens of $4 \mathrm{x}$ and an aperture value setting equal to $N . A .=0.13$. The light source is a D-LH $12 \mathrm{~V}-100 \mathrm{~W}$ halogen lamp and all the experiments were performed in a dark room in order to maintain a constant level of luminosity with time and to minimize any reflections and shadows. The images were 
collected by using a high-speed camera having a monochrome sensor; for the investigated flow regimes, a resolution of $920 \times 2048$ pixels and a frame rate up to 387 frames/s were employed. The exposure time of each image is equal to $\delta T=10^{-6} \mathrm{~s}$, thus three order of magnitude smaller than the sampling frequency. The interaction of light with the fully dissolved dye molecules was verified to obey the Lambert-Beer's law stating that the absorbance is linear with the concentration; hence flow visualization images were processed off-line to convert pixel intensities into normalized depth-averaged dye concentration images. Details on the calibration and image analysis procedures can be found in [17], together with a more detailed description of the whole experimental set up.

\section{NUMERICAL SIMULATIONS}

The liquid motion is described by the unsteady incompressible non-dimensional form of the Navier-Stokes equations (omitted here for the sake of brevity). The hydraulic diameter of the outlet channel, $d$, is used as a reference length, while the reference velocity is the bulk velocity, $U$, which is constant throughout the mixer. Therefore, the Reynolds number is defined as $R e=\rho U d / \mu$ where $\rho$ and $\mu$ are the density and viscosity of the operating liquid. The Navier-Stokes equations are coupled with a transport equation governing the dye dynamics, which is written in the following non-dimensional form:

$$
\frac{\partial \phi}{\partial \theta}+\mathbf{u} \cdot \nabla \phi=\frac{1}{P e} \nabla^{2} \phi,
$$

where $\phi$ is the dye mass fraction, $\theta$ is the non-dimensional time, $\mathbf{u}$ the non-dimensional fluid velocity and $P e=U d / D$ is the Peclet number, with $D$ denoting the dye molecular diffusivity. It is worth noting that for liquids the Schmidt number is very large, i.e. $S c=$ $\mu /(\rho D)=O\left(10^{3} \div 10^{4}\right)$, so that $P e=S c R e=O\left(10^{6}\right)$.

The NEK5000 open-source code [6], based on a high-order spectral-element method, was employed to carry out the numerical simulations presented herein. The computational domain is discretized by means of hexahedral elements and the velocity space in each element is spanned by $N$ th-order Lagrange polynomial interpolants, based on tensor-product arrays of Gauss-Lobatto-Legendre (GLL) quadrature points. Time discretization is carried out by means of a third order backward differential formula. The diffusive terms are treated implicitly, while a third order explicit extrapolation is considered for the convective terms. 
For the present simulations with $\alpha=20^{\circ}$, the computational domain is the same as the experimental model, except for the length of the inlet channels, which is lower, i.e. $L_{i}=6.875 d$. Hence, a unidirectional fully developed velocity profile is set at the inlets (see [1] for more details). The length of the mixing channel, $L_{o}=25 d$ is also lower than that of the experimental model. Free outflow conditions are used at the outlet, by assuming zero normal gradient of the velocity components in the $x$ and $z$ directions and zero normal stresses. The sensitivity analysis carried out in [1] for a T-mixer geometry indicated that the adopted value of $L_{o}$ together with the free outflow boundary conditions are adequate to avoid significant spurious effects of domain truncation. No-slip boundary conditions are finally imposed at the mixer walls. The computational grid consists of 15680 spectral elements, with a uniform element size of $0.075 \mathrm{~d}$ in both directions of the cross-sections of the mixer channels while along their axis the size varies between $0.08 d$ and $0.5 d$. The polynomial order $N$ for the velocity is fixed to 6 , while an order of 4 is adopted for the pressure, thus using the common $P_{N} / P_{N-2}$ scheme, for a total of about $10^{7}$ degrees of freedom for each velocity component. The time step is equal to $7.0 \cdot 10^{-4} \frac{d}{U}$, corresponding to a CFL number $\leq 0.3$. This computational set up is practically the same as that used in the simulations in [26] for a arrow-shaped micro-mixer having a different aspect ratio. Arrow-shaped mixers having $\alpha=10^{\circ}, 15^{\circ}$ and $25^{\circ}$ have also been simulated. The computational set-up is the same as for $\alpha=20^{\circ}$.

\section{RESULTS AND DISCUSSION}

The experimental and numerical results obtained for different Reynolds number are presented in this section. In the following figures, all coordinates are made non-dimensional by using the mixing channel hydraulic diameter, i.e. $X=x / d, Y=y / d$ and $Z=z / d$. The local dye concentration is normalized by the inlet concentration so that it ranges from 0 to 1. As for the dye concentration equation, in numerical simulations, stability issues forced to use a rather low $S c$ number, i.e. $S c=O(100)$, as in [5]. However, as shown in [10], such low $S c$ number does not affect the results in the confluence region analyzed in the present work, due to the presence of strong transversal convection. The numerical data were post-

processed on purpose to average the concentration field over the mixer depth, thus allowing the proper comparison with the experimental flow visualizations. More details are given in 


\section{A. Vortex regime}

Let us start by analyzing the results obtained at $R e=120$. Figure 2a shows the steady depth-averaged dye concentration field from experimental flow visualizations, while the corresponding numerical concentration field is reported in Figure 2b. The agreement between experimental flow visualizations and numerical simulations is very good and, in both cases, at this low Reynolds number the two flow streams appear to remain segregated in the mixing channel. The three dimensional vortical structures characterizing this regime are visualized in Figure 2c by means of the $\lambda_{2}$ criterion [12]. According to this criterion, a vortex is defined as a connected fluid region where the second largest eigenvalue, $\lambda_{2}$, of the symmetric tensor $\mathbf{L}=\mathbf{S} \cdot \mathbf{S}+\mathbf{A} \cdot \mathbf{A}$ is negative, where $\mathbf{S}$ and $\mathbf{A}$ indicate the symmetric and anti-symmetric part of the velocity gradient, that are the strain rate and vorticity tensors, respectively. The isosurface of $\lambda_{2}$ in Figure 2c is 3D; for ease of comparison, the same 2D view as for the dye field visualizations is shown in the bottom panel, while a top view is provided in the top panel. The top parts of these structures are strictly related to the flow recirculations occurring near the top wall of the mixer at the confluence of the two streams entering the inlet channels; these recirculations can be clearly seen in Figure 3a. In more details, part of the flow at the confluence of the streams is lifted up near the central plane of the mixer by the velocity induced by the top parts of the vortical structures; this leads to a saddle point in the streamlines in the $x-y$ plane (see Figure 3a). The lifted up fluid is in part directly convected downstream at the lateral edges of the vortex heads, closing in this way the recirculating regions, again shown in Figure 3a. However, part of it flows along the vortical structures and eventually is convected downstream in the mixing channel only when reaching the vicinity of the mixer walls parallel to the $x-y$ plane (see also Figure $3 \mathrm{~b}$ ). This is in turn connected with the formation of two pairs of counter-rotating legs for each vortical structure (see also Figure 4). For low Reynolds numbers, as the present one, the flow and the described vortices preserve the two reflectional geometrical symmetries in the whole domain and, in particular, also in the mixing channel (see Figure 4). Consequently, convection does not help mixing, which occurs only through diffusion, and, as a result, the two streams remain segregated also in the mixing channel (Figure 4c). This scenario is 
typical of the vortex regime and, as found also in [26], it is qualitatively the same as the one observed and widely characterized in the literature for T-mixers (see e.g. [1, 4]).

\section{B. Engulfment regime}

As the Reynolds number increases, the flow in the mixer enter the so-called engulfment regime, as shown, for instance, in Figures 5, 6 and 7 at $R e=140$. The flow is still steady, but, looking at the concentration fields in Figures $5 \mathrm{a}$ and $5 \mathrm{~b}$ it is clear how the flow patterns in this regime are more complex and how the mixing between the two streams is largely increased compared to the vortex regime. The agreement between numerical simulations and experimental flow visualizations is once again very good.

Figure 6 shows the flow features in the top part of the mixer and, namely, the same quantities as in Figure 3. A first observation is that the recirculation regions move closer to the top wall and to each other and become smaller as the Reynolds number increases. The same is for the top parts of the 3D vortical structures, which are tightly connected with the flow recirculations. More striking is the rupture of the double mirror symmetry of the flow. Indeed, it can be seen that the recirculation zones and the top parts of the vortical structures are tilted with respect to the $z$ direction. As in the vortex regime, a fraction of the fluid entering the top part of the mixer, where inclined vortical structures are present, flows along them towards the side walls, before being convected downstream. However, now there is a preferential direction, which is that corresponding to the lower deviation angle. As a consequence, the amount of fluid which flows downstream at the two edges of each structure is significantly different and hence, the two legs entering the outflow channel are not equal in terms of intensity, shape, and position. As can be better seen in Figure 7, moving towards the end of the mixing channel, the weakest couple of legs disappears and only two main co-rotating vortices survive, which are further fed by the flow entering the channel directly below the recirculation regions. The signature of these complex vorticity structures can be identified in the dye concentration fields in Figures 5a and 5b: for instance, the two recirculation regions near the top walls are clearly visible and they appear slightly superimposed because of their tilting. Also the strong vortex legs in the mixing channel are visible, while in the first part, roughly up to $Y=-1.9$ also the effects of the weakest

legs can be identified. The previously described vorticity pattern is in agreement with the 
results of [26] and, once again, it is qualitatively the same as the one observed and widely characterized in the literature for T-mixers (see e.g. $[1,4]$ ). The main difference respect to T-mixers is that the onset of the engulfment regime occurs at lower Reynolds numbers, as found in [26]: indeed, in the present case, the critical Reynolds number for the onset of the engulfment regime is found, both experimentally and numerically, to be between 125 and 130, while for a T-mixer having the same geometry of the channels and operating in the same conditions, the engulfment was found to establish for Re between 140 and 145 [17]. It is well known that mixing in the engulfment regime, thanks to the help of convection, is much larger than in the vortex one. This can be qualitatively appreciated by comparing Figures $4 \mathrm{c}$ and $7 \mathrm{c}$ and it will be better quantified in Section IV C. The dye distribution in the mixing channel cross-sections is only available in numerical simulations, because the experiments only give the concentration averaged through the channel depth. Thus we compared the depth-averaged concentration obtained at different sections throughout the mixing channel to the experimental one and the agreement was found to be very good (not shown here for the sake of brevity). From a practical viewpoint, the lower value of the Reynolds number at which the engulfment regime occurs makes the arrow-shape interesting.

Let us now further increase the Reynolds number. Figure 8 shows the steady dye concentration field and vortical structures at $R e=150$, while the main flow features in the top part of the mixer are highlighted in Figure 9. Starting from the dye concentration field, once again the agreement between experiments and numerical simulations is very good. The concentration field patterns in the mixing channel are qualitatively the same as at $R e=140$, while the signatures of the flow recirculation regions in the top part of the mixer are now only hardly visible. This is because the recirculation zones further move towards the top wall compared to $R e=140$ and become smaller and weaker. The same is for the top parts of the vortical structures, which in the present case appear to be disconnected from their legs. Also their tilting angle increases with Reynolds, consistently with what observed for T-mixers $([1,4,17])$. Apart from these differences, the flow pattern in the top part of the mixer is qualitatively the same as for $R e=140$ with most of the fluid flowing along the vortex heads in the direction corresponding to the smaller deviation angle and then being convected downwards in the mixing channel. As a result, two main co-rotating vortex legs are present in the mixing channel as at $R e=140$ (Figure 10). Compared with the case at $R e=140$, these two co-rotating vortical structures tend to become closer to each other and 
eventually to merge in the mixing channel (compare Figs. 10 and 7).

Moving to $R e=170$ (Figures 11, 12,13), the flow is still steady but a gradual change in the flow topology can be noticed. The recirculation regions near the top wall have almost

disappeared and consequently also the top parts of the vortical structures. This in turn implies that there is no more fluid lifted up in the center of the mixer; conversely, some of the fluid reaching the top part of the mixer undergoes a sort of spiral pattern near the center of the mixing channel and it is convected downward in this way. Such spiral pattern promotes the immediate merging, through clinging, of the two co-rotating vortical structures in the mixing channel. This leads to the presence of a single vortical leg in the mixing channel, as also shown in Figure 13. This type of flow pattern is not found in T-mixers, in which the flow remains in the classical engulfment regime for Reynolds numbers up to $R e \approx 220$ and then passes to an unsteady periodic behavior (see e.g. $[1,3,4,8,10]$ ). Finally, it can be inferred from the dye field sections in Figure 13 that the new flow topology is less efficient in promoting mixing than the classical engulfment one (compare e.g. with Figure 10). However, this will be quantified and discussed in Section IV C.

If we keep increasing the Reynolds number, the central vortical structure in the mixing channel increases in size and intensity (see e.g. Figures 14, and 15 and 16 for $R e=190$ ). The dye distribution in different cross-sections of the mixing channel well indicates that the strength of the central vortical structure leads to a spiral pattern, which is more effective in promoting mixing than for the $R e=170$ case.

Finally, for $R e \geq 200$ the flow becomes unsteady and periodic; the unsteady regimes will be the object of future investigations.

\section{Mixing performance}

To quantify mixing between the two streams, following [7], we use the mixing degree, $\delta_{m}$, defined as follows:

$$
\delta_{m}(Y)=1-\frac{\sigma_{b}(Y)}{\sigma_{\max }}
$$

where $\sigma_{b}$ is the standard deviation of the volumetric flow given by the following expression:

$$
\sigma_{b}^{2}(Y)=\frac{\int\left(\phi(X, Y, Z)-\bar{\phi}_{b}\right)^{2} u_{y}(X, Y, Z) d X d Z}{\int u_{y}(X, Y, Z) d X d Z}
$$


where $u_{y}(X, Y, Z)$ is the $y$-velocity component in the $X-Z$ cross-section of the mixing channel and the bulk or cup mixing average $\bar{\phi}_{b}$ is

$$
\bar{\phi}_{b}=\frac{\int \phi(X, Y, Z) u_{y}(X, Y, Z) d X d Z}{\int u_{y}(X, Y, Z) d X d Z}
$$

Finally, $\sigma_{\max }$ is the maximum value of $\sigma_{b}$, which is achieved when the two streams remain completely segregated, and it is defined as:

$$
\sigma_{\max }=\sqrt{\bar{\phi}_{b}\left(1-\bar{\phi}_{b}\right)}
$$

As a result, $\delta_{m}$ is bounded in the range $0 \leq \delta_{m} \leq 1, \delta_{m}=0$ and $\delta_{m}=1$ indicating a completely segregated and a fully mixed flow, respectively.

Figure 17 shows $\delta_{m}$ computed in numerical simulations at the $Y=-8$ section of the mixing channel as a function of the Reynolds number. The curve obtained for numerical simulations carried out with the same code for a T-mixer having the same geometry of the channels and operating in the same conditions [10] is also reported for comparison. Only Reynolds numbers at which the flow is steady are shown in Figure 17; by further increasing Re unsteady periodic regimes take place (see e.g. $[1,5,17]$ for T-mixers).

Figure 17 confirms and quantifies the previously made observations. A first sharp increase of the mixing degree can be seen at the onset of the engulfment regime, which, as previously said, occurs at lower Reynolds numbers for the arrow-shaped mixer than for the T-mixer. By further increasing the Reynolds number, in the arrow-shaped case, $\delta_{m}$ initially increases up to a local maximum approximately at $R e=140$ and then drops reaching a local minimum close to $R e=170$. This decrease of $\delta_{m}$ is due to the previously described flow topology changes leading to the formation of a single vortical structure in the mixing channel, whose induced velocity field is less efficient than the two distinct co-rotating vortices present in the classical engulfment regime. Then, mixing increases again because this vortical structure expands in size and increases its intensity (see $R e=190$ ). At approximately $R e=200$ the vortical structure starts to oscillate and the flow becomes unsteady and periodic in time; hence this $R e$ is not reported in the figure. As previously said, the unsteady regimes will be investigated in details in future works.

It is interesting to note that the behavior of $\delta_{m}$ in the steady engulfment regime for the T-mixer is instead monotonically increasing with $R e$ and, despite the earlier increase of the mixing degree, there is a range of Reynolds numbers in which $\delta_{m}$ is lower in the arrow-shaped 
case. This suggests that a more accurate control of the operating conditions is needed for a arrow-shaped mixer than for a T-mixer, in order to obtain optimal mixing also within the engulfment regime.

\section{Sensitivity to the tilting angle of the inlet channels}

We finally analyze the sensitivity to $\alpha$ by means of additional simulations. Let us start with the behavior of the degree of mixing as a function of the Reynolds number, shown in Figure 18. The results for the T-mixer and for $\alpha=20^{\circ}$, previously shown in Figure 17, are also reported here for comparison. As expected on the basis of the results in [26], the Reynolds number at which the engulfment regime and the corresponding sudden increase of mixing take place, progressively decreases as $\alpha$ becomes larger. For $\alpha=10^{\circ}$ and $15^{\circ}$, the degree of mixing monotonically increases with $R e$ throughout the whole engulfment regime, as for T-mixers. The topology of the flow is indeed practically the same as for T-mixers, with the persistence of the top parts of the $3 \mathrm{D}$ vortical structures near the upper walls of the mixer and of the two co-rotating stronger legs in the mixing channel for the whole engulfment regime. This can be seen, for instance, in Figure 19 showing a isosurface of the $\lambda_{2}$ vortex indicator for $\alpha=10^{\circ}$ at $R e=180$ and 200. The $\lambda_{2}$ topology in the engulfment

regime for T-mixers can be found, for instance, in [4], [1], [17] and [10]. Conversely, as for the previously analyzed configuration with $\alpha=20^{\circ}$, the mixer with $\alpha=25^{\circ}$ is characterized by a non monotonic behavior of the degree of mixing with Re. Also in this case, the decrease of mixing occurring at approximately $R e=125$ is due to the same change in the flow topology as described in Sec. IV B, i.e. the disappearance of the top parts of the vortical structures and the consequent formation of a unique vortical structure in the mixing channel (see Figure 20), which is less efficient in promoting mixing.

\section{CONCLUDING REMARKS}

The effect of Reynolds number on the liquid mixing in a arrow-mixer has been analyzed by direct numerical simulations and flow visualizations. Although the latter did not used any confocal system, the signature of the flow regimes was well evident from the ex- 
perimental images. Moreover a proper comparison was possible between predictions and measurements, through the definition of post-processing routines based on the evaluation of depth-averaged concentration maps. In all cases the agreement between numerical results and flow visualizations was very good.

The engulfment regime was found to take place at a lower Reynolds number than for a T-mixer having the same cross-section and it was accompanied to a sharp improvement of the degree of mixing. However, further increasing the Reynolds number, a reduction of the mixing performance was observed. Thanks to the availability of flow and vorticity fields from direct numerical simulations, it was ascertained that this is related to a deep change of the flow topology. In particular, the recirculation regions near the top of the mixer were found to move closer to the top wall and to become weaker as the Reynolds number increases. At $R e=170$ they completely disappear, with the liquid reaching the top part of the mixer undergoing a spiral pattern near the center of the mixing channel and being convected downward. This leads to the formation of a strong vortical leg in the mixing channel, constituted by the clinging of the two co-rotating vortical structures, that is less efficient in promoting mixing with respect to the classical engulfment regime, where these two co-rotating vortices remain instead separated. Indeed, the minimum of the mixer performance in the engulfment regime was found to take place at about $R e=170$, with a mixing degree that was approximately half of the one at $R e=140$. In particular the degree of mixing was here even lower than the T-mixer one at the same Re. Further increasing the $R e$, the mixing degree augments again, because of the increase in size and strength of the unique vortical structure in the mixing channel. Finally, at $R e=200$ this structure starts to oscillate leading to an unsteady periodic flow. The sensitivity to the tilting angle has also been analyzed by means of numerical simulations. As expected, the value of the Reynolds number at which there is the onset of the engulfment regime and then sudden increase of mixing becomes progressively lower with increasing $\alpha$. For low tilting angles $\left(\alpha=10^{\circ}\right.$ and $15^{\circ}$ ) the degree of mixing monotonically increases with $R e$ throughout the whole steady engulfment regime, as for T-mixers. Conversely, for $\alpha=25^{\circ}$ the behavior of the degree of mixing in non monotonic with $R e$, as for $\alpha=20^{\circ}$, with a sudden decrease of mixing due to the same change in the flow topology as that observed for $\alpha=20^{\circ}$.

Thus, arrow-mixers are interesting because they trigger mixing at lower $R e$ compared to T-mixers and this effect is more pronounced for larger tilting angles of the inlet channels. 
However, when the angle exceeds some critical value, which for the present configuration is between $\alpha=15^{\circ}$ and $20^{\circ}$, the degree of mixing is no more increasing monotonically with $R e$; therefore, a careful control of the operating conditions is needed for these configurations.

\section{ACKNOWLEDGMENTS}

This work was supported by the University of Pisa through the "Progetti di Ricerca di Ateneo PRA 2017-2018"funding program. The authors wish also to thank CINECA computing center (Bologna, Italy) for allowance of computational resources under ISCRA program (class B project "MIRE"). Moreover the authors are grateful to Deborah Spaltro, Tommaso Pannuzi, Matteo Antognoli and Cesare Merello.

[1] Andreussi, T., Galletti, C., Mauri, R., Camarri, S. \& Salvetti, M.V. 2015 Flow regimes in T-shaped micro-mixers. Computers and Chemical Engineering 76, 150-159.

[2] Babu, H.V., Satu, S., Haderlein, M., Peukert, W. \& Verma, N. 2016 Numerical investigation of flow patterns and concentration profiles in y-mixers. Chemical Engineering and Technology 39 (10), 1963-1971.

[3] Dreher, S., Kockmann, N. \& Woias, P. 2009 Characterization of laminar transient flow regimes and mixing in T-shaped micromixers. Heat Transfer Engineering 30 (1-2), 91-100.

[4] Fani, A., Camarri, S. \& Salvetti, M.V. 2013 Investigation of the steady engulfment regime in a three-dimensional T-mixer. Physics of Fluids 25 (6).

[5] Fani, A., Camarri, S. \& Salvetti, M.V. 2014 Unsteady asymmetric engulfment regime in a T-mixer. Physics of Fluids 26 (7).

[6] Fischer, P.F., Lottes, J.W. \& Kerkemeier, S.G. 2008 nek5000 web page. Web page: http://nek5000. mcs. anl. gov .

[7] Galletti, C., Arcolini, G., Brunazzi, E. \& Mauri, R. 2015 Mixing of binary fluids with composition-dependent viscosity in a T-shaped micro-device. Chemical Engineering Science 123, 300-310.

[8] Galletti, C., Brunazzi, E. \& Mauri, R. 2017 Unsteady mixing of binary liquid mixtures with composition-dependent viscosity. Chemical Engineering Science 164, 333-343. 
[9] Galletti, C., Brunazzi, E., Siconolfi, L., Spaltro, D. \& Mauri, R. 2017 Mixing performance of arrow-shaped micro-devices. Chemical Engineering Transactions 57, 13091314 .

[10] Galletti, C., Mariotti, A., Siconolfi, L., Mauri, R. \& Brunazzi, E. 2019 Numerical investigation of flow regimes in T-shaped micromixers: benchmark between finite volume and spectral element methods. Canadian Journal of Chemical Engineering 97, 528-541.

[11] Galletti, C., Roudgar, M., Brunazzi, E. \& Mauri, R. 2012 Effect of inlet conditions on the engulfment pattern in a T-shaped micro-mixer. Chemical Engineering Journal 185-186, $300-313$.

[12] Jeong, J. \& Hussain, F. 1995 On the identification of a vortex. Journal of Fluid Mechanics 285, 6994 .

[13] Kockmann, N., Kiefer, T., Engler, M. \& Woias, P. 2006 Convective mixing and chemical reactions in microchannels with high flow rates. Sensors and Actuators B: Chemical $117(2), 495-508$.

[14] Kockmann, N. \& Roberge, D. M. 2011 Transitional flow and related transport phenomena in curved microchannels. Heat Transfer Engineering 32 (7-8), 595-608.

[15] Kumar, V., Paraschivoiu, M. \& Nigam, K. D. P. 2011 Single-phase fluid flow and mixing in microchannels. Chemical Engineering Science 66 (7), 1329-1373.

[16] Lee, C.-Y., Wang, W.-T., Liu, C.-C. \& Fu, L.-M. 2016 Passive mixers in microfluidic systems: a review. Chemical Engineering Journal 288, 146 - 160.

[17] Mariotti, A., Galletti, C., Mauri, R., Salvetti, M.V. \& Brunazzi, E. 2018 Steady and unsteady regimes in a T-shaped micro-mixer: Synergic experimental and numerical investigation. Chemical Engineering Journal 341, 414-431.

[18] Orsi, G., Galletti, C., Brunazzi, E. \& Mauri, R. 2013 Mixing of two miscible liquids in T-shaped microdevices. Chemical Engineering Transactions 32, 1471-1476.

[19] Poole, R. J., Alfateh, M. \& Gauntlett, A. P. 2013 Bifurcation in a T-channel junction: Effects of aspect ratio and shear-thinning. Chemical Engineering Science 104, 839-848.

[20] Rahimi, M., Azimi, N., Parsamogadam, M.A., Rahimi, A. \& Masahy, M.M. 2017 Mixing performance of $\mathrm{t}, \mathrm{y}$, and oriented $\mathrm{y}$-micromixers with spatially arranged outlet channel: evaluation with villermaux/dushman test reaction. Microsystem Technologies 23 (8), 31173130. 
[21] Reddy Cherlo, S. K. \& Pushpavanam, S. 2010 Effect of depth on onset of engulfment in rectangular micro-channels. Chemical Engineering Science 65 (24), 6486-6490.

[22] Roberge, D. M., Ducry, L., Bieler, N., Cretton, P. \& Zimmermann, B. 2005 Microreactor technology: a revolution for the fine chemical and pharmaceutical industries? Chemical Engineering and Technology 28 (3), 318-323.

[23] Rossetti, I. \& Compagnoni, M. 2016 Chemical reaction engineering, process design and scale-up issues at the frontier of synthesis: Flow chemistry. Chemical Engineering Journal 296, $56-70$.

[24] Salvetti, M.V., Mariotti, A., Galletti, C. \& Brunazzi, E. 2018 Steady and unsteady regimes in a T-shaped micro-mixer: Synergic experimental and numerical investigation. in Proceedings of the ASME 2018 5th Joint US-European Fluids Engineering Division Summer Meeting, FEDSM2018 July 15-20, 2018, Montreal, Quebec, Canada, Publisher: American Society of Mechanical Engineers, Fluids Engineering Division, Code 1411023.

[25] Siconolfi, L., Camarri, S. \& Salvetti, M.V. 2018 T-mixer operating with water at different temperatures: Simulation and stability analysis. Physical Review Fluids 3 (3).

[26] Siconolfi, L., Fani, A., Camarri, S. \& Salvetti, M.V. 2016 Effect of geometry modifications on the engulfment in micromixers: Numerical simulations and stability analysis. European Journal of Mechanics - B/Fluids 55 (2), 360 - 366.

[27] Soleymani, A., Yousefi, H. \& Turunen, I. 2008 Dimensionless number for identification of flow patterns inside a T-micromixer. Chemical Engineering Science 63 (21), 5291-5297.

[28] Yao, X., Zhang, Y., Du, L., Liu, J. \& YaO, J. 2015 Review of the applications of microreactors. Renewable and Sustainable Energy Reviews 47, 519-539.

[29] You, B.J., Choi, Y. \& Im, S.G. 2017 Influence of adjusting the inlet channel confluence angle on mixing behaviour in inertial microfluidic mixers. Microfluid Nanofluid 21 (7), 121. 


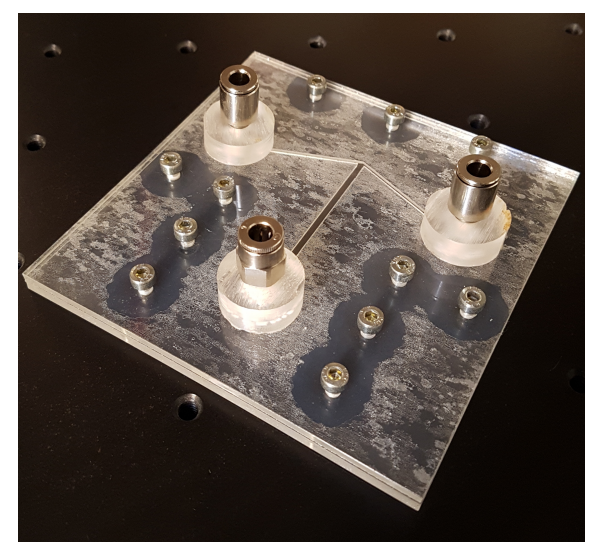

(a)

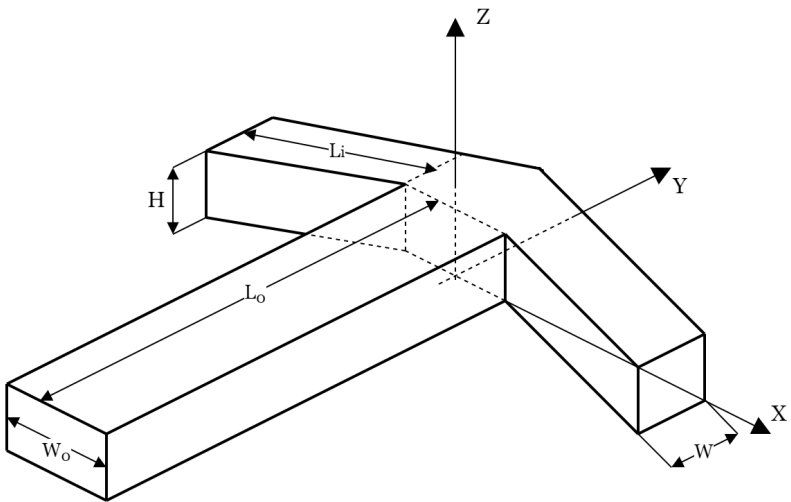

(b)

Figure 1. Experimental model (a) and sketch of the arrow-mixer with $\alpha=20^{\circ}$ (b).

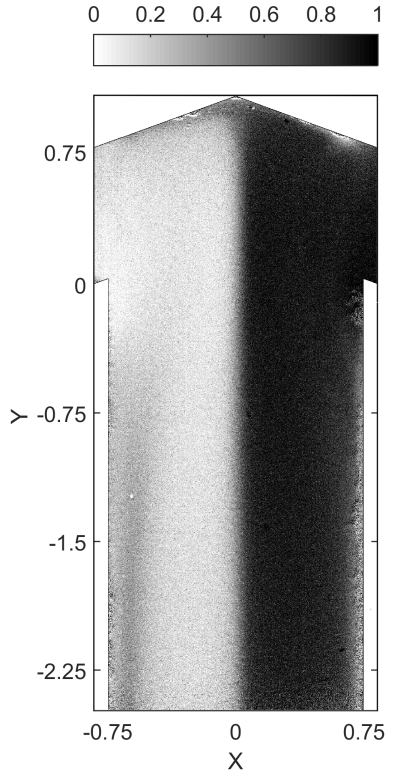

(a)

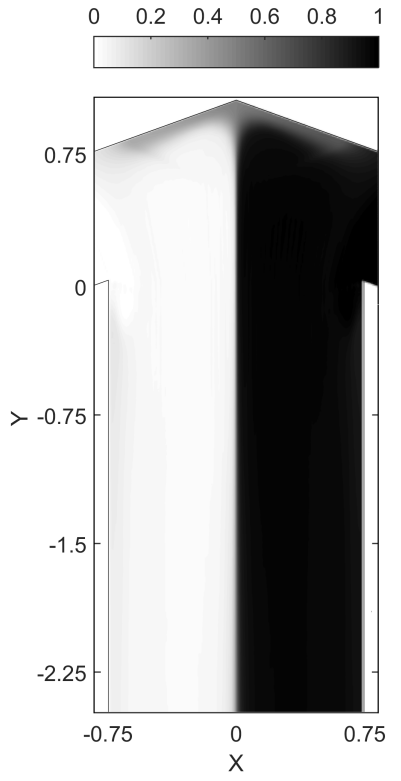

(b)
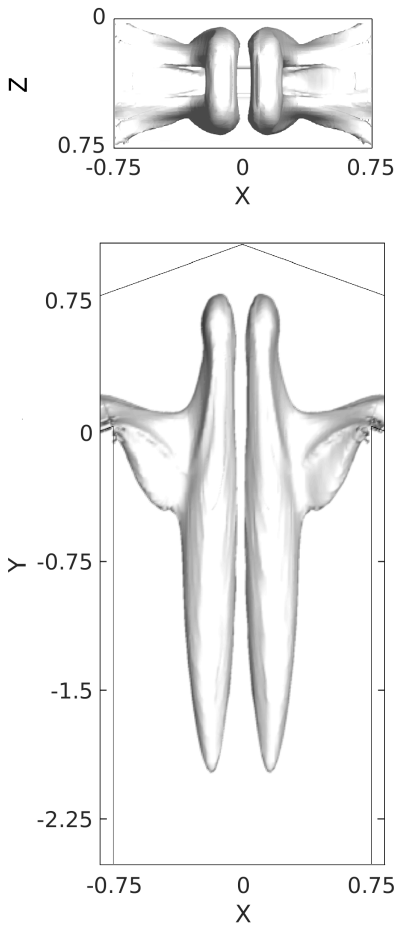

(c)

Figure 2. Experimental (a) and numerical (b) dye concentration fields and isosurface of the $\lambda_{2}$ vortex indicator (c) for the arrow-mixer with $\alpha=20^{\circ}$ at $R e=120$. 

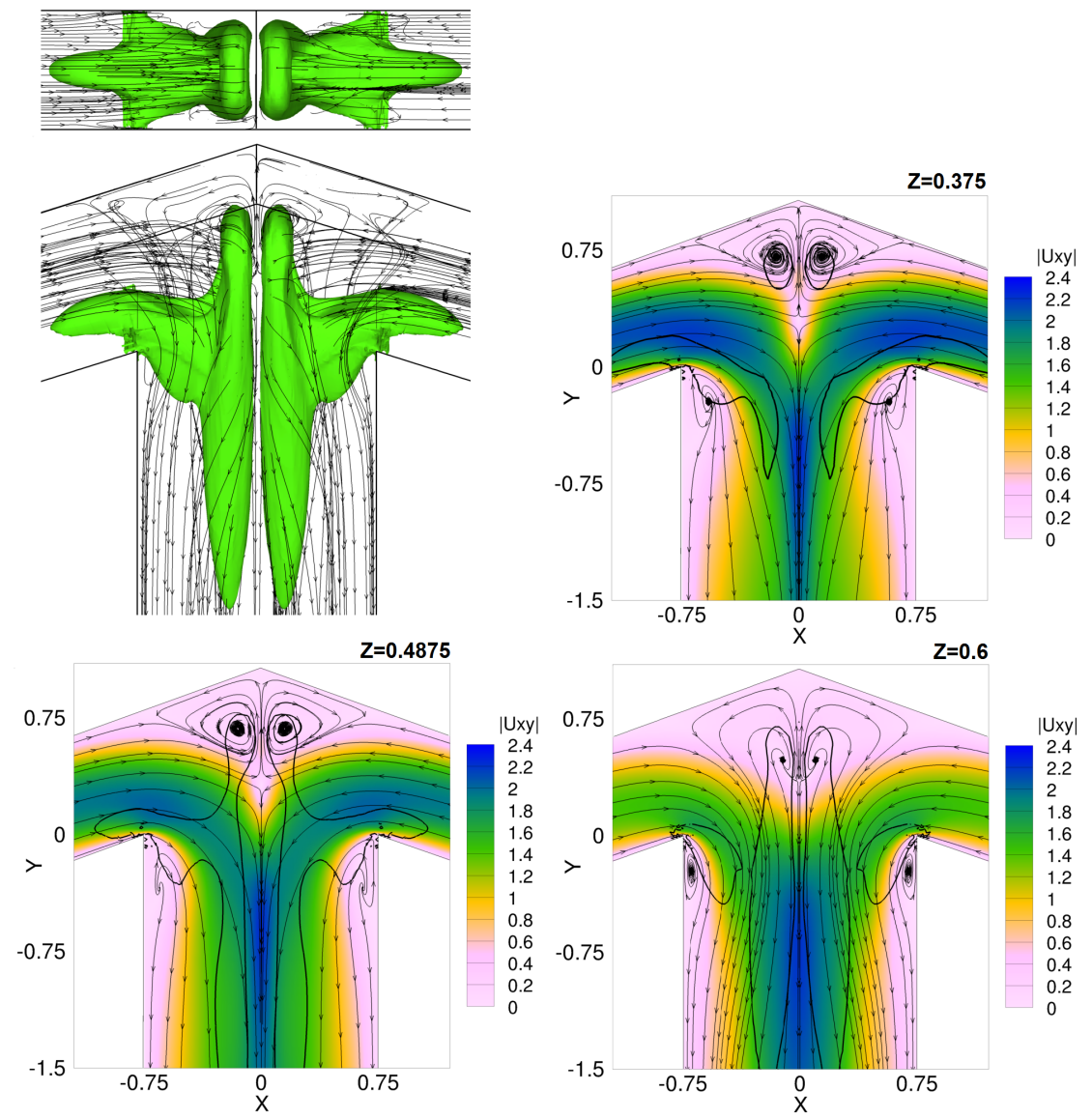

(a)
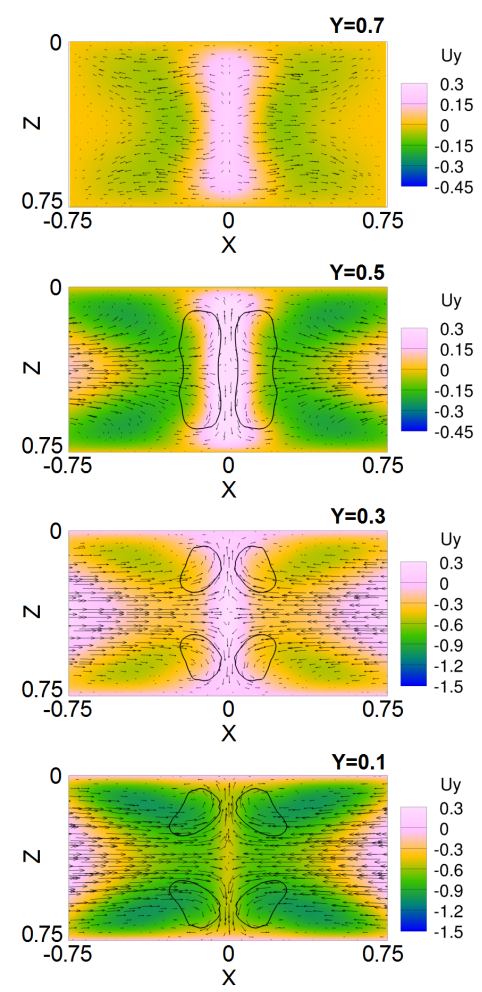

(b)

Figure 3. Flow features in the top part of the arrow-mixer with $\alpha=20^{\circ}$ at $R e=120$ : (a) Isosurface of the $\lambda_{2}$ vortex indicator and velocity streamlines (top left), contours of the in-plane velocity magnitude, velocity streamlines and trace of the isosurface of the $\lambda_{2}$ (thick line) for the sections at $Z=0.375$ (top right), $Z=0.4875$ (bottom left) and $Z=0.6$ (bottom right); (b) contours of the $y$-velocity, in-plane velocity vectors and trace of the isosurface of the $\lambda_{2}$ (thick line) for the cross-sections at (from top to bottom) $Y=0.7, Y=0.5, Y=0.3$ and $Y=0.1$. 


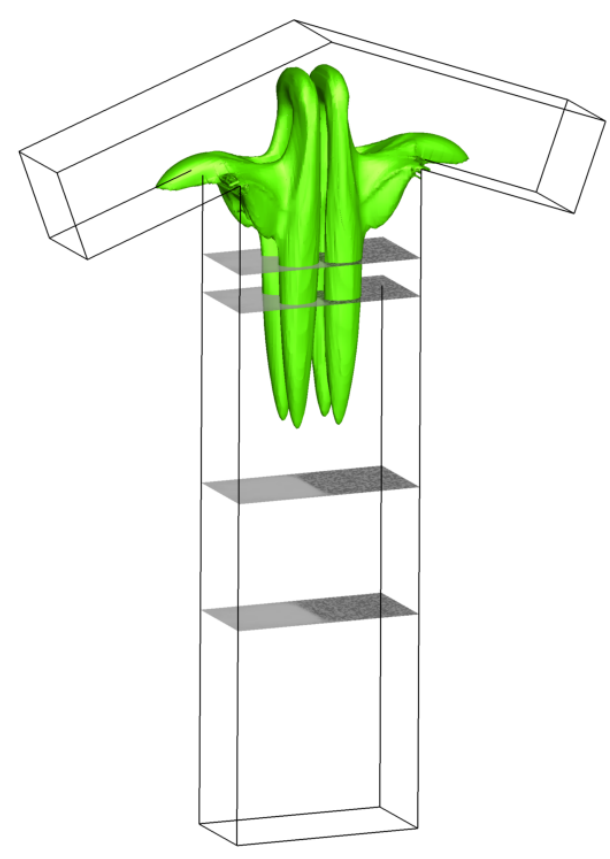

(a)
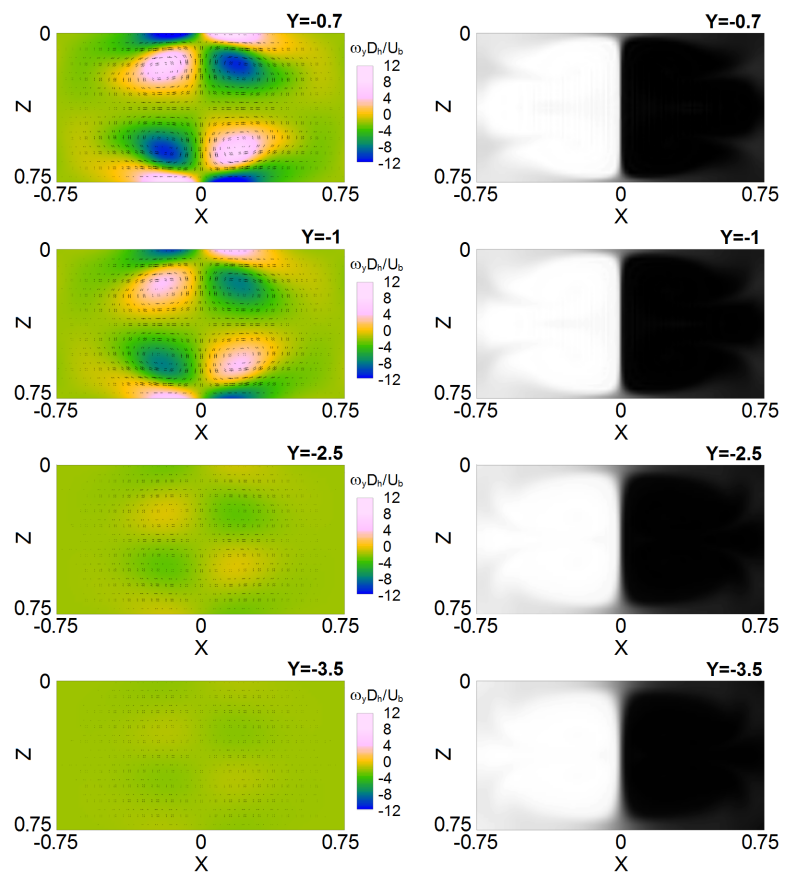

(b)

(c)

Figure 4. Isosurface of the $\lambda_{2}$ vortex indicator from a different view (a), $y$-vorticity and in-plane velocity (b) and dye concentration fields (c) for the arrow-mixer with $\alpha=20^{\circ}$ at $R e=120$. Considered cross-sections (from top to bottom): $Y=-0.7, Y=-1, Y=-2.5$ and $Y=-3.5$. All the results are from numerical simulations. 


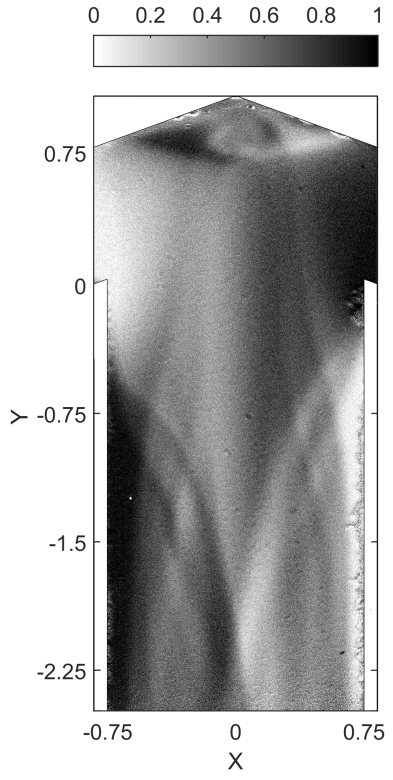

(a)

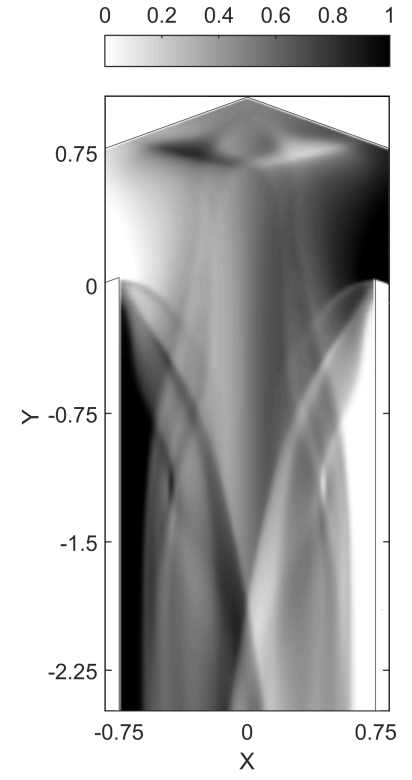

(b)
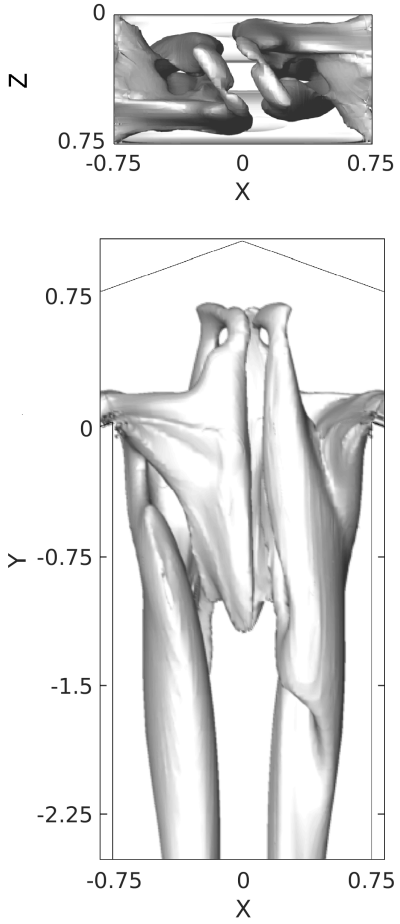

(c)

Figure 5. Experimental (a) and numerical (b) dye concentration fields and isosurface of the $\lambda_{2}$ vortex indicator (c) for the arrow-mixer with $\alpha=20^{\circ}$ at $R e=140$. 


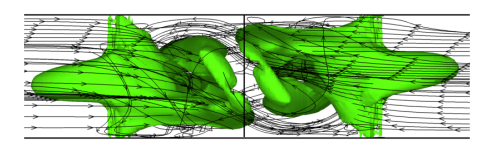

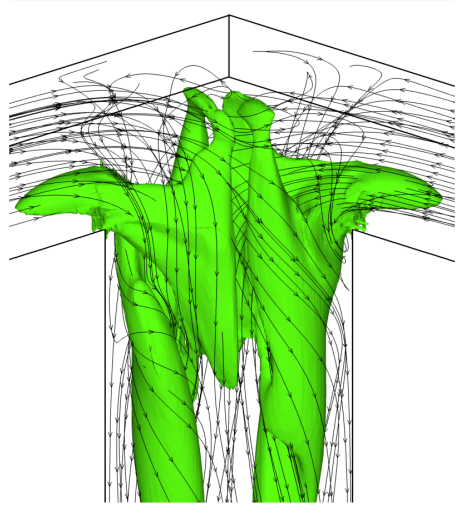

$Z=0.4875$
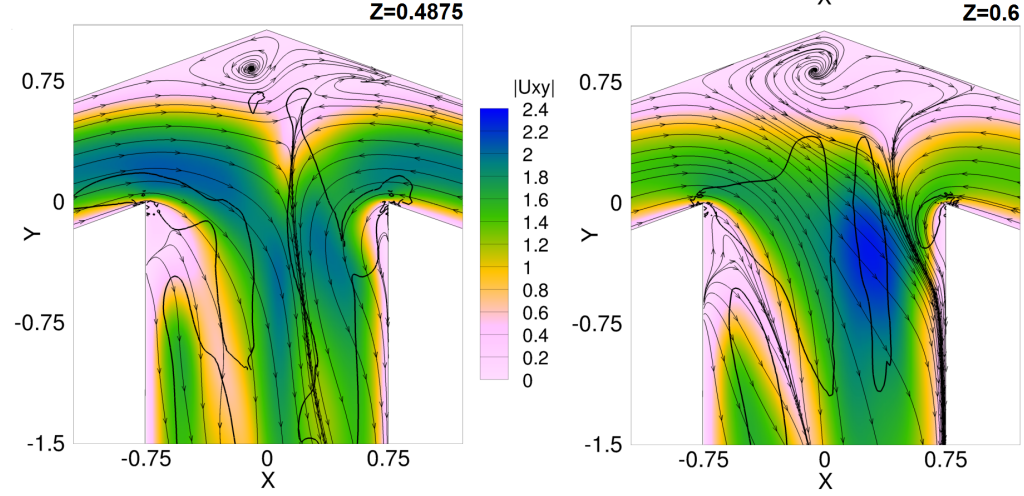

(a)
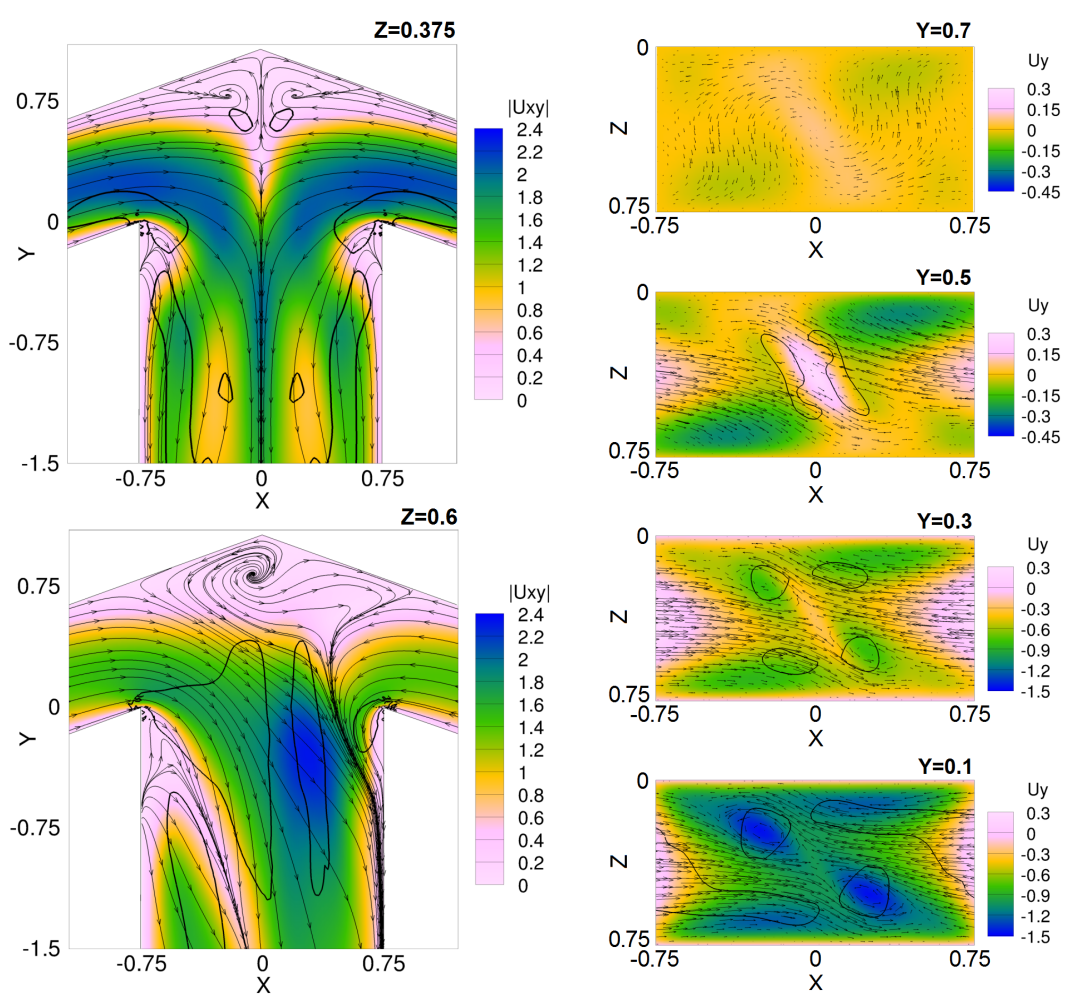

(b)

Figure 6. Flow features in the top part of the arrow-mixer with $\alpha=20^{\circ}$ at $R e=140$ : (a) Isosurface of the $\lambda_{2}$ vortex indicator and velocity streamlines (top left), contours of the in-plane velocity magnitude, velocity streamlines and trace of the isosurface of the $\lambda_{2}$ (thick line) for the sections at $Z=0.375$ (top right), $Z=0.4875$ (bottom left) and $Z=0.6$ (bottom right); (b) contours of the $y$-velocity, in-plane velocity vectors and trace of the isosurface of the $\lambda_{2}$ (thick line) for the cross-sections at (from top to bottom) $Y=0.7, Y=0.5, Y=0.3$ and $Y=0.1$. 


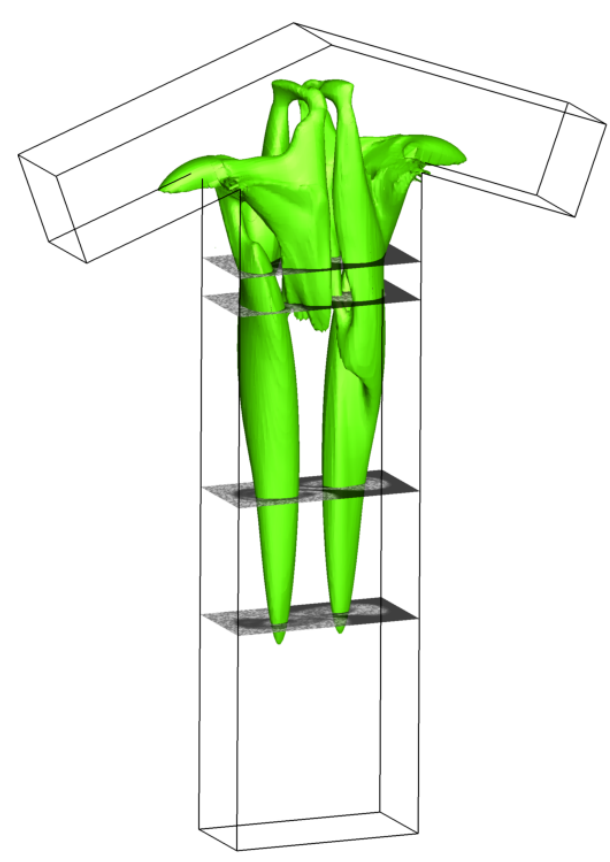

(a)
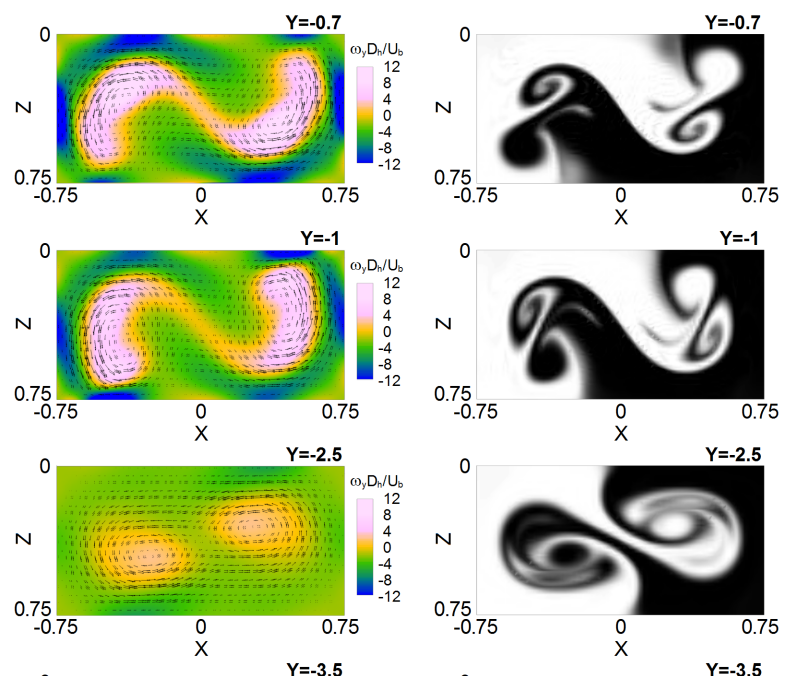

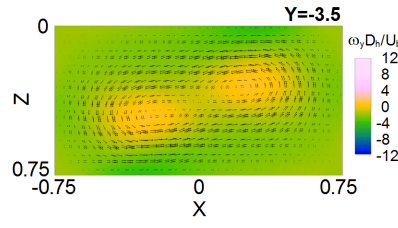

(b)

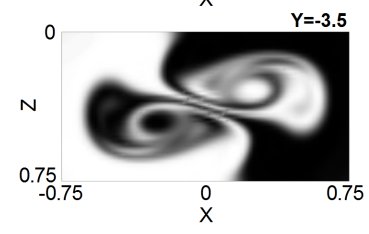

(c)

Figure 7. Isosurface of the $\lambda_{2}$ vortex indicator (a) $y$-vorticity fields (b) and dye concentration fields (c) for the arrow-mixer with $\alpha=20^{\circ}$ at $R e=140$. Considered cross-sections (from top to bottom): $Y=-0.7, Y=-1, Y=-2.5$ and $Y=-3.5$. All the results are from numerical simulations. 


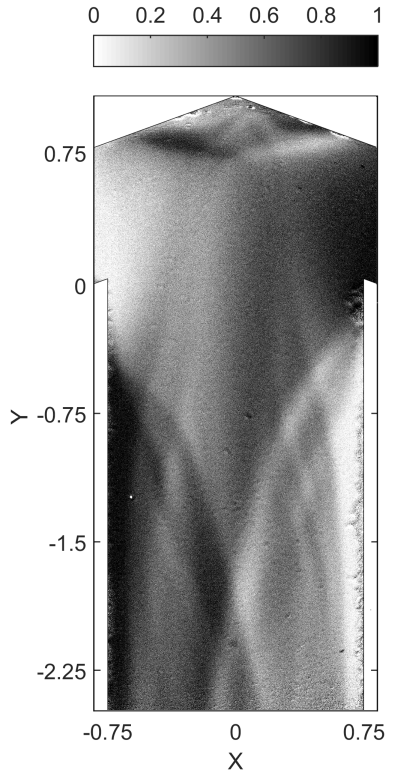

(a)

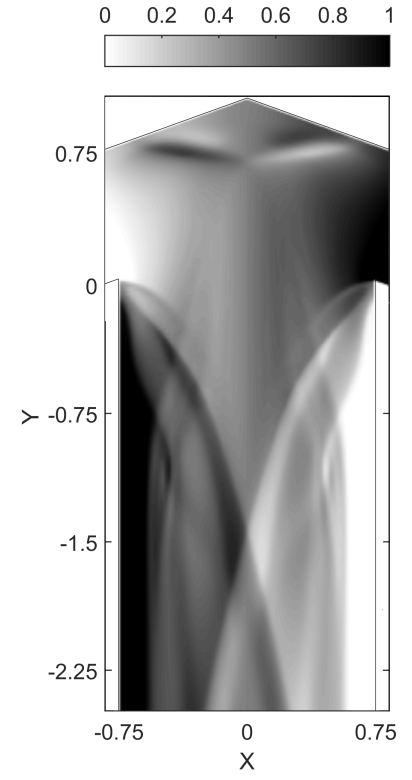

(b)
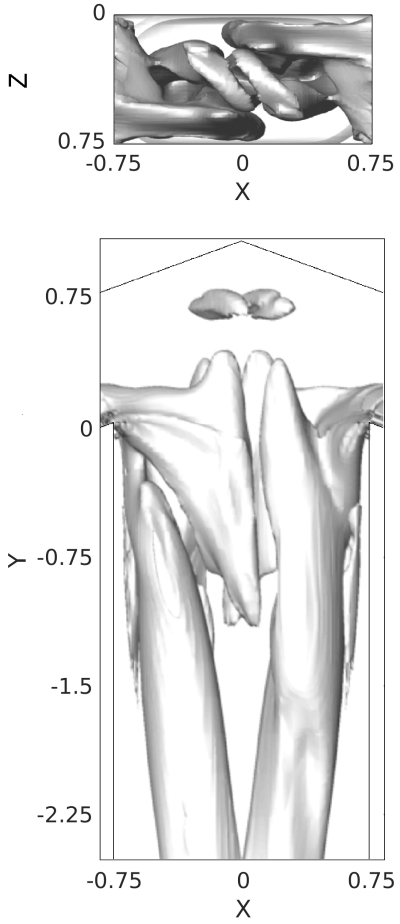

(c)

Figure 8. Experimental (a) and numerical (b) dye concentration fields and isosurface of the $\lambda_{2}$ vortex indicator (c) for the arrow-mixer with $\alpha=20^{\circ}$ at $R e=150$. 

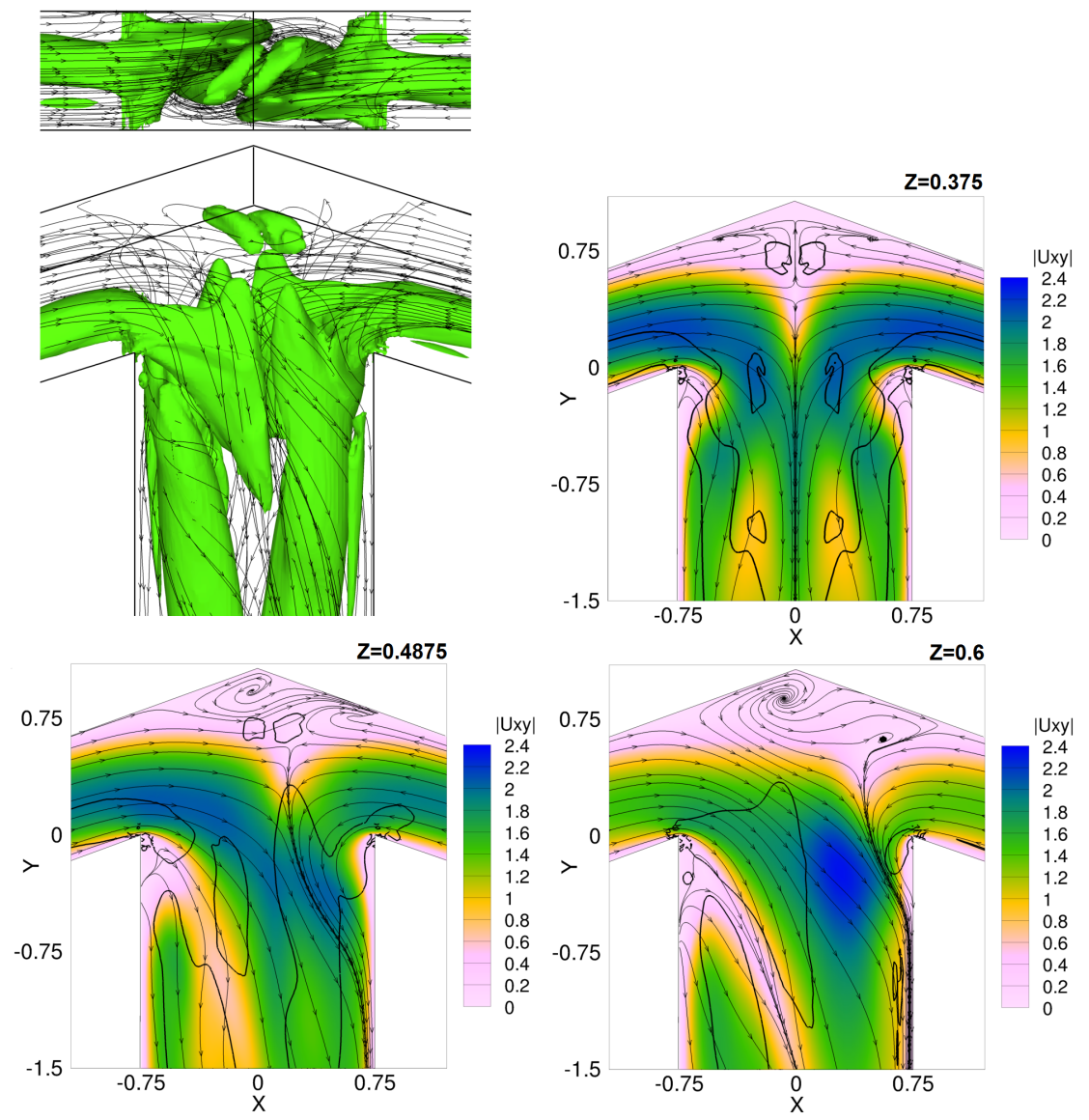

(a)
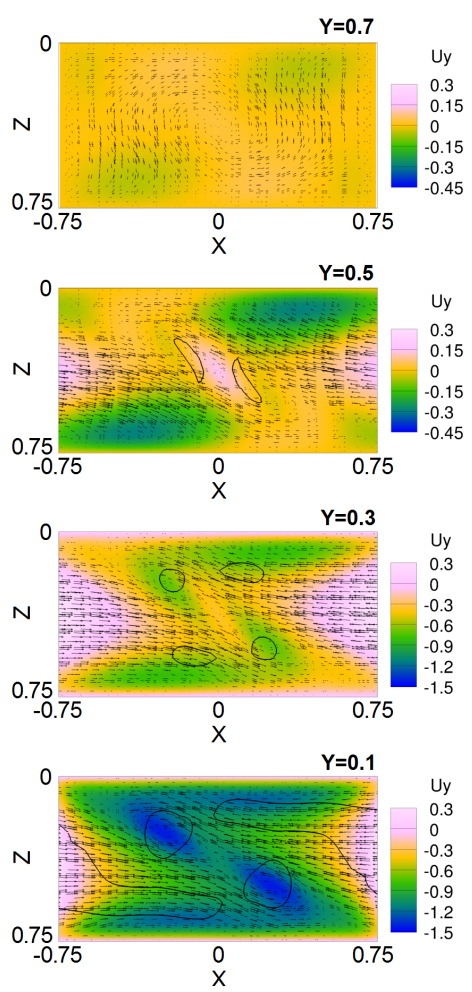

(b)

Figure 9. Flow features in the top part of the arrow-mixer with $\alpha=20^{\circ}$ at $R e=150$ : (a) Isosurface of the $\lambda_{2}$ vortex indicator and velocity streamlines (top left), contours of the in-plane velocity magnitude, velocity streamlines and trace of the isosurface of the $\lambda_{2}$ (thick line) for the sections at $Z=0.375$ (top right), $Z=0.4875$ (bottom left) and $Z=0.6$ (bottom right); (b) contours of the $y$-velocity, in-plane velocity vectors and trace of the isosurface of the $\lambda_{2}$ (thick line) for the cross-sections at (from top to bottom) $Y=0.7, Y=0.5, Y=0.3$ and $Y=0.1$. 


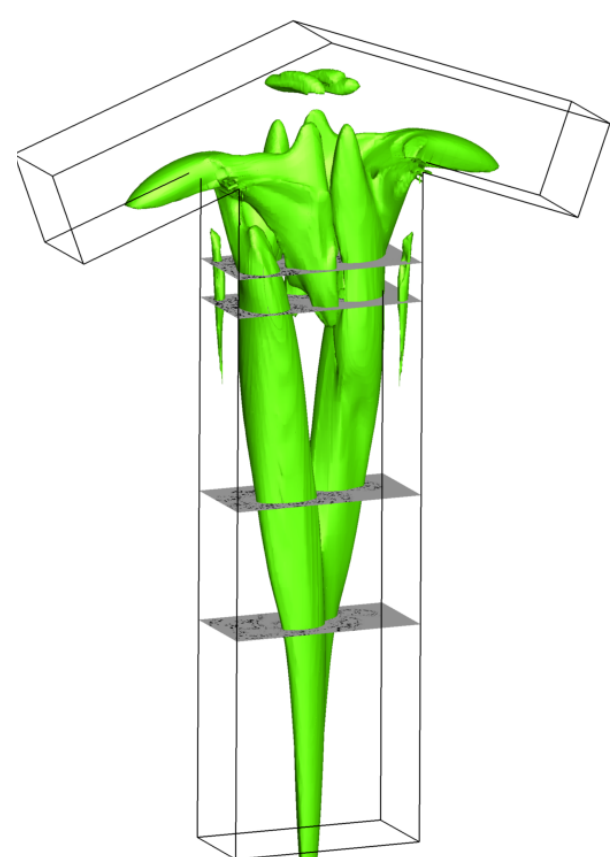

(a)
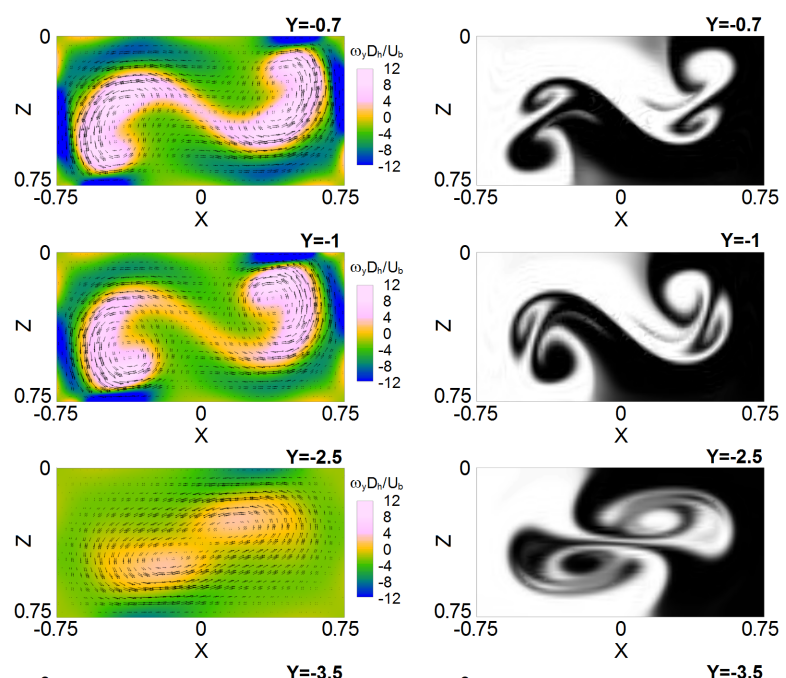

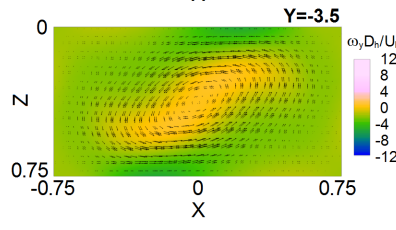

(b)

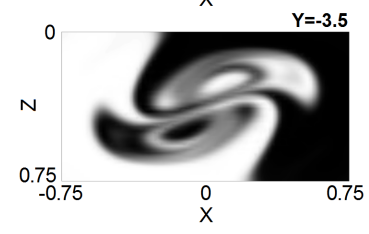

(c)

Figure 10. Isosurface of the $\lambda_{2}$ vortex indicator (a) $y$-vorticity fields (b) and dye concentration fields (c) for the arrow-mixer with $\alpha=20^{\circ}$ at $R e=150$. Considered cross-sections (from top to bottom): $Y=-0.7, Y=-1, Y=-2.5$ and $Y=-3.5$. All the results are from numerical simulations. 


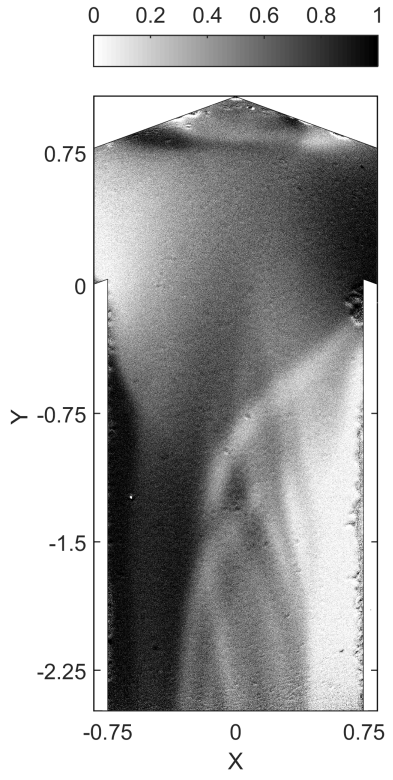

(a)

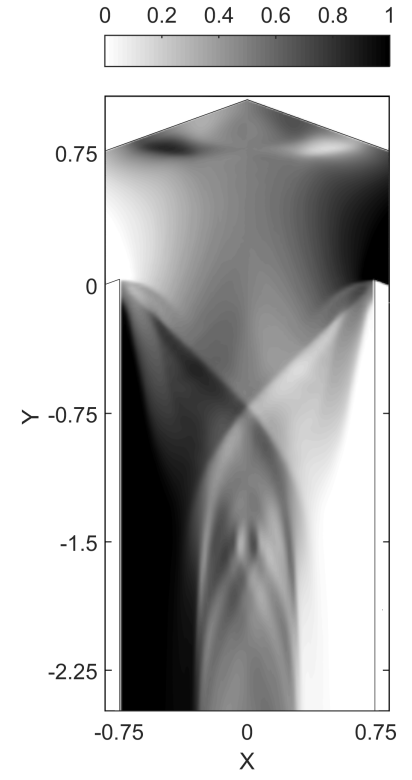

(b)
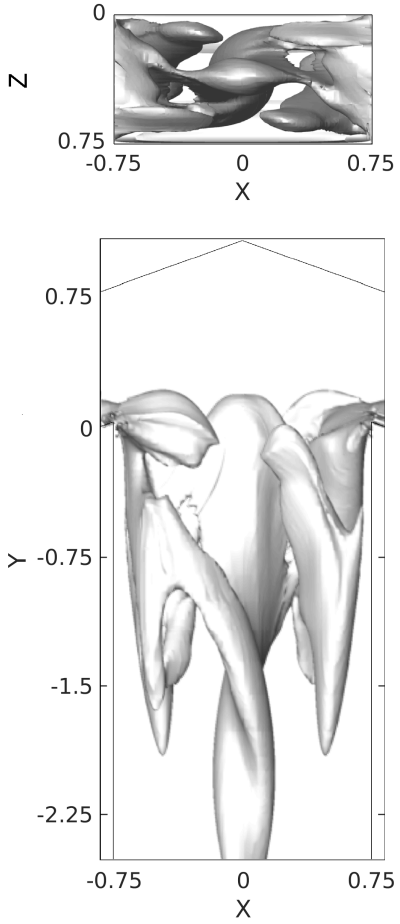

(c)

Figure 11. Experimental (a) and numerical (b) dye concentration fields and isosurface of the $\lambda_{2}$ vortex indicator (c) for the arrow-mixer with $\alpha=20^{\circ}$ at $R e=170$. 


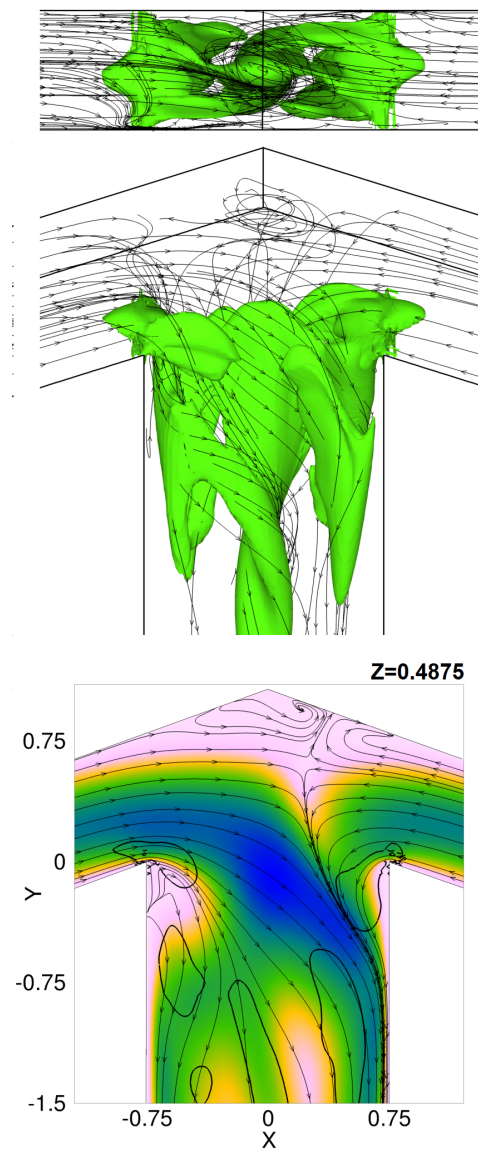

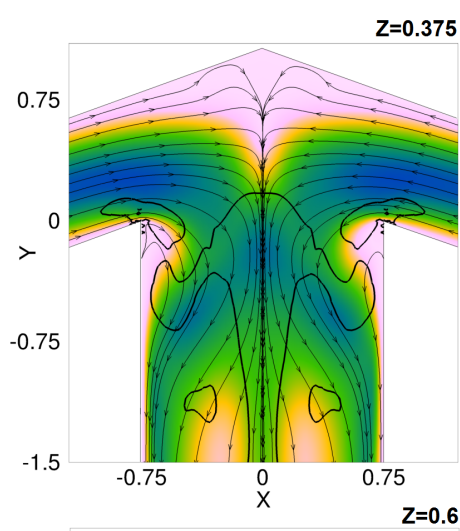

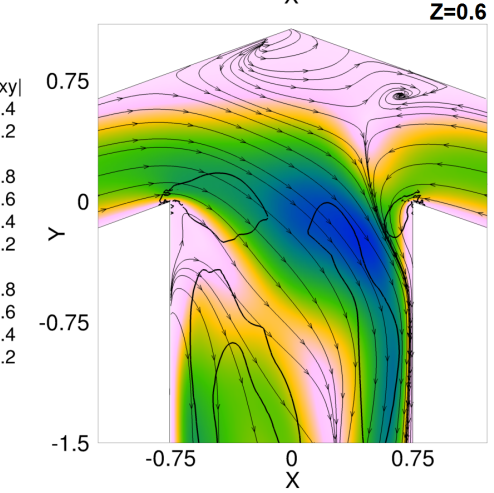

(a)
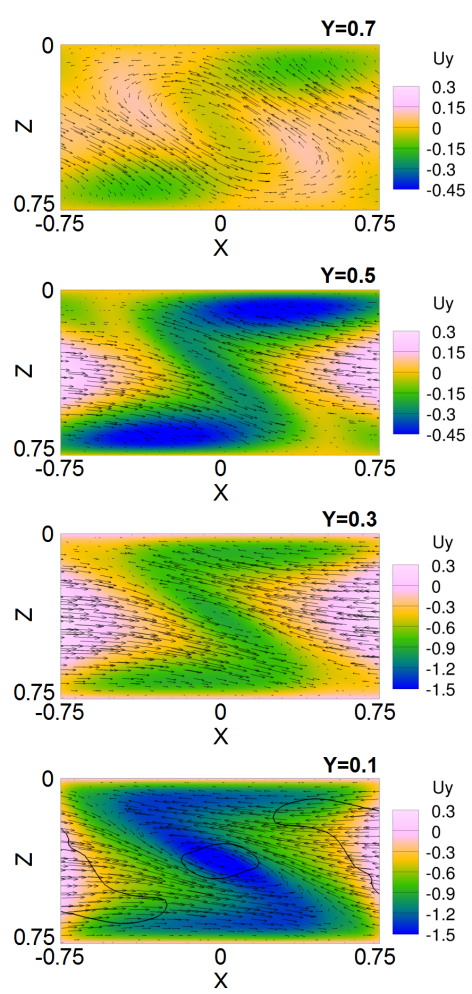

(b)

Figure 12. Flow features in the top part of the arrow-mixer with $\alpha=20^{\circ}$ at $R e=170$ : (a) Isosurface of the $\lambda_{2}$ vortex indicator and velocity streamlines (top left), contours of the in-plane velocity magnitude, velocity streamlines and trace of the isosurface of the $\lambda_{2}$ (thick line) for the sections at $Z=0.375$ (top right), $Z=0.4875$ (bottom left) and $Z=0.6$ (bottom right); (b) contours of the $y$-velocity, in-plane velocity vectors and trace of the isosurface of the $\lambda_{2}$ (thick line) for the cross-sections at (from top to bottom) $Y=0.7, Y=0.5, Y=0.3$ and $Y=0.1$. 


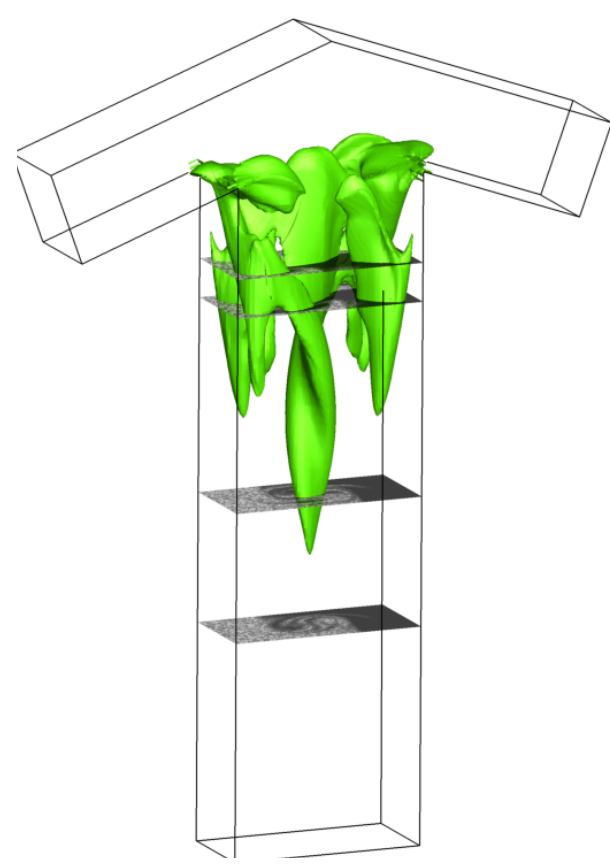

(a)
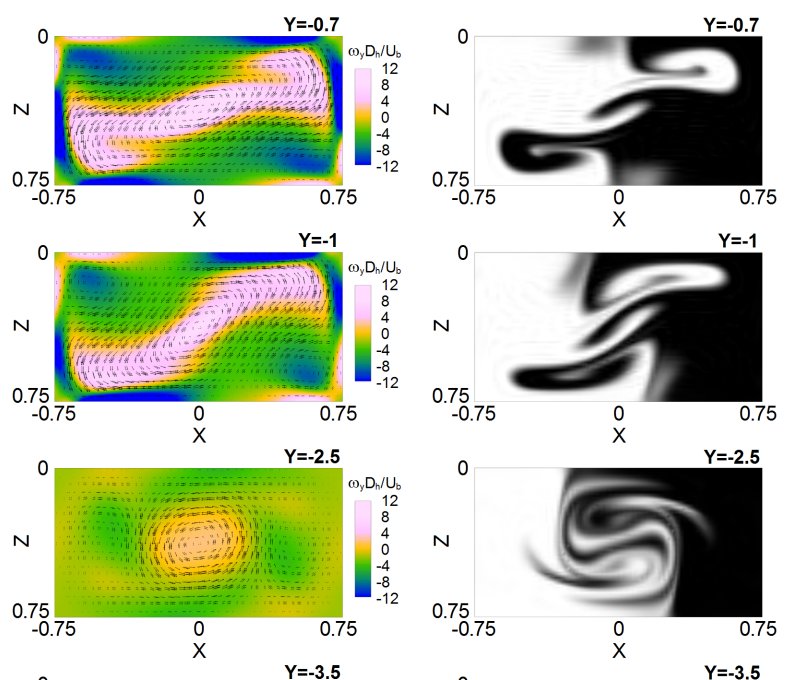

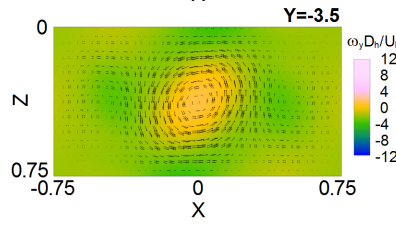

(b)

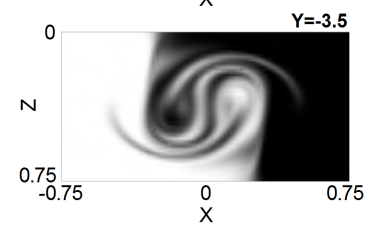

(c)

Figure 13. Isosurface of the $\lambda_{2}$ vortex indicator (a) $y$-vorticity fields (b) and dye concentration fields (c) at $R e=170$. Considered cross-sections (from top to bottom): $Y=-0.7, Y=-1$, $Y=-2.5$ and $Y=-3.5$. All the results are from numerical simulations. 


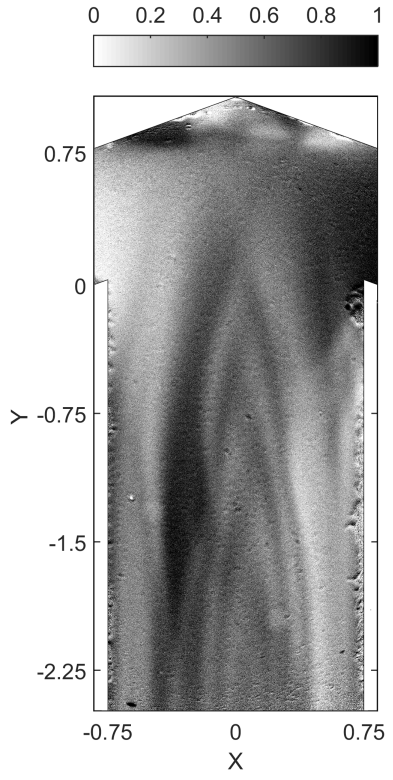

(a)

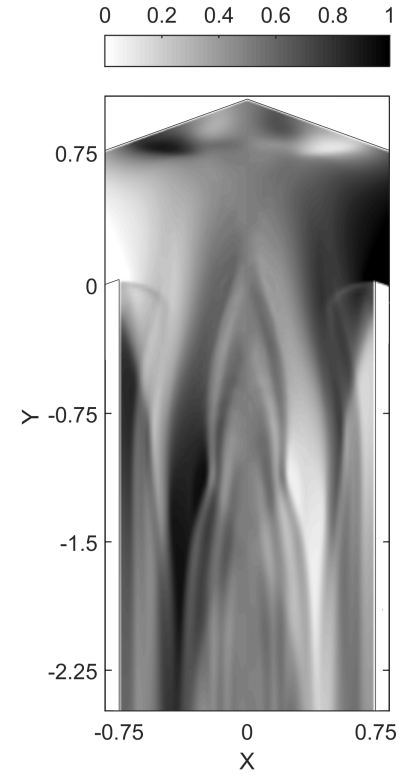

(b)
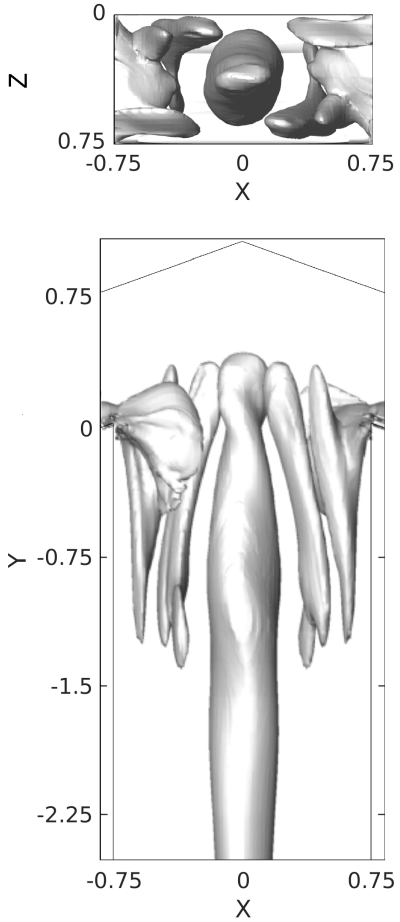

(c)

Figure 14. Experimental (a) and numerical (b) dye concentration fields and isosurface of the $\lambda_{2}$ vortex indicator (c) for the arrow-mixer with $\alpha=20^{\circ}$ at $R e=190$. 


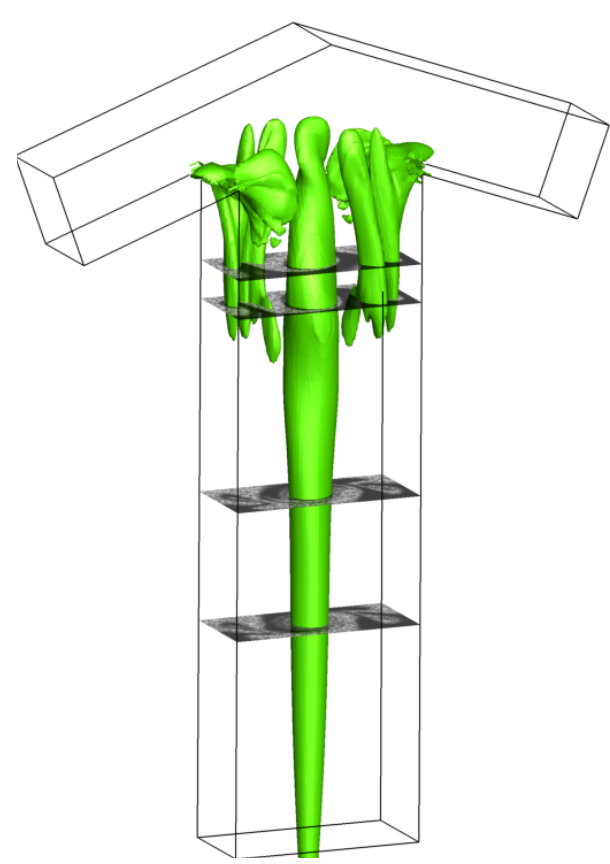

(a)
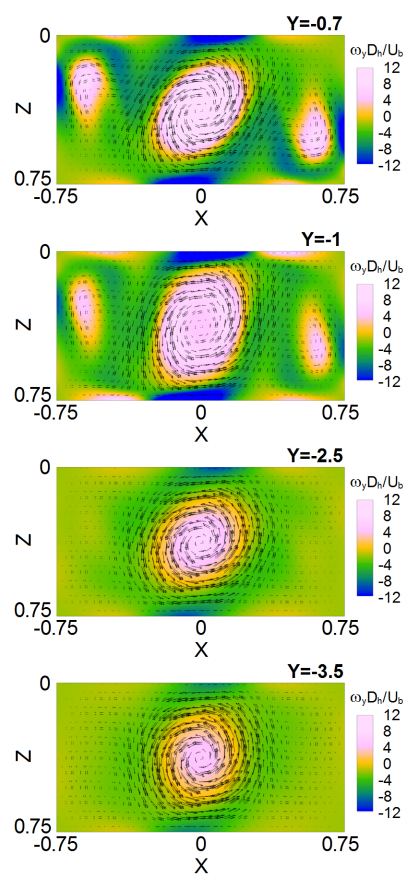

(b)
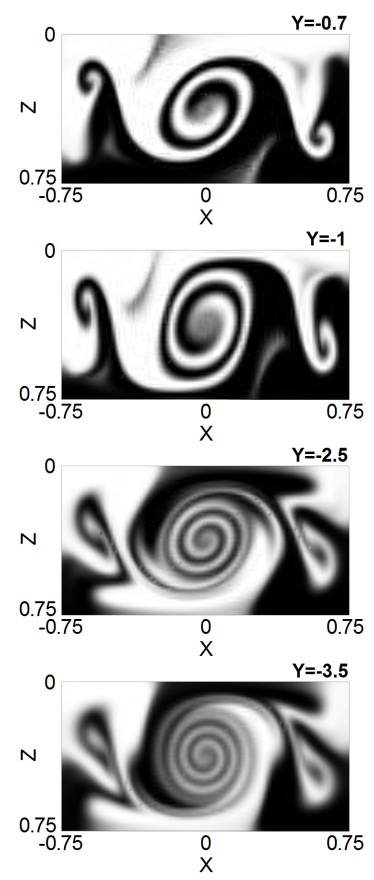

(c)

Figure 15. Isosurface of the $\lambda_{2}$ vortex indicator (a) $y$-vorticity fields (b) and dye concentration fields (c) for the arrow-mixer with $\alpha=20^{\circ}$ at $R e=190$. Considered cross-sections (from top to bottom): $Y=-0.7, Y=-1, Y=-2.5$ and $Y=-3.5$. All the results are from numerical simulations. 


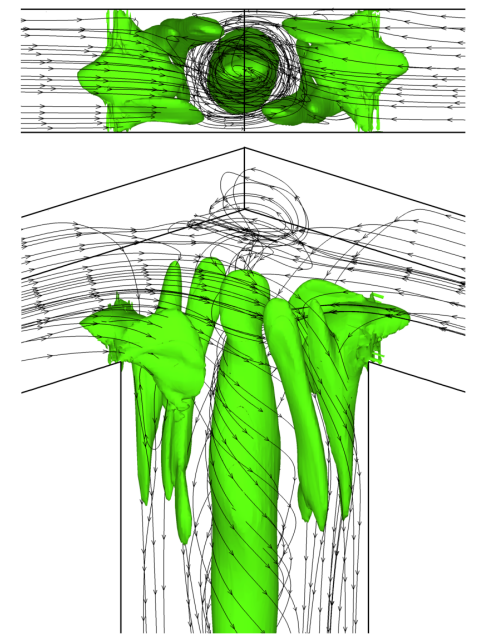

$\mathrm{Z}=\mathbf{0 . 4 8 7 5}$
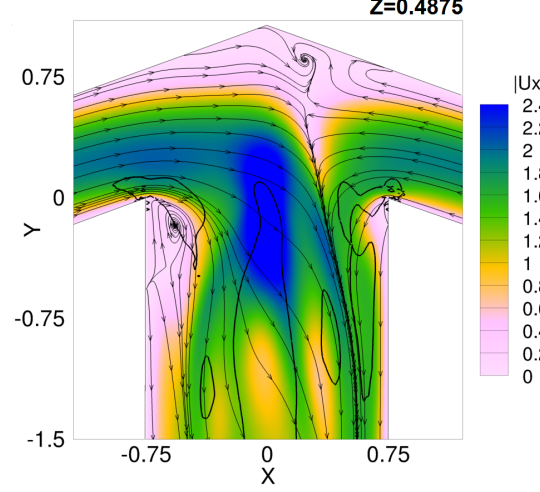
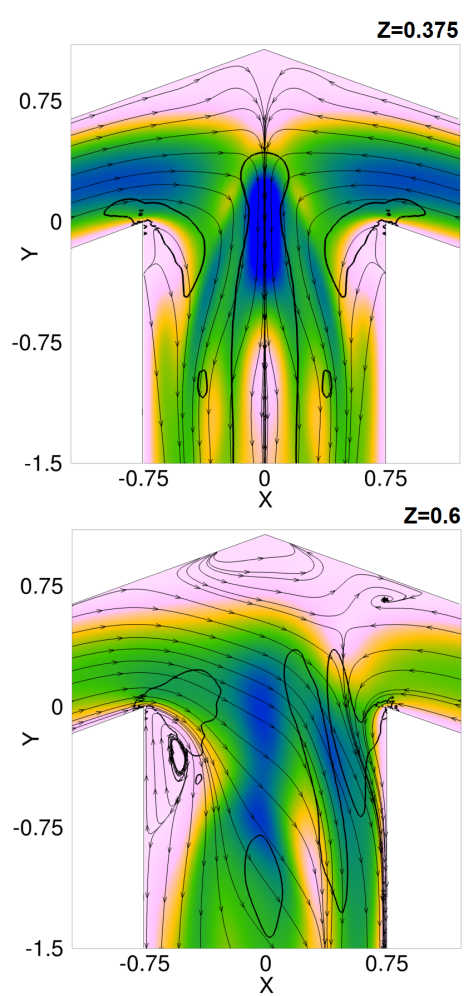

(a)
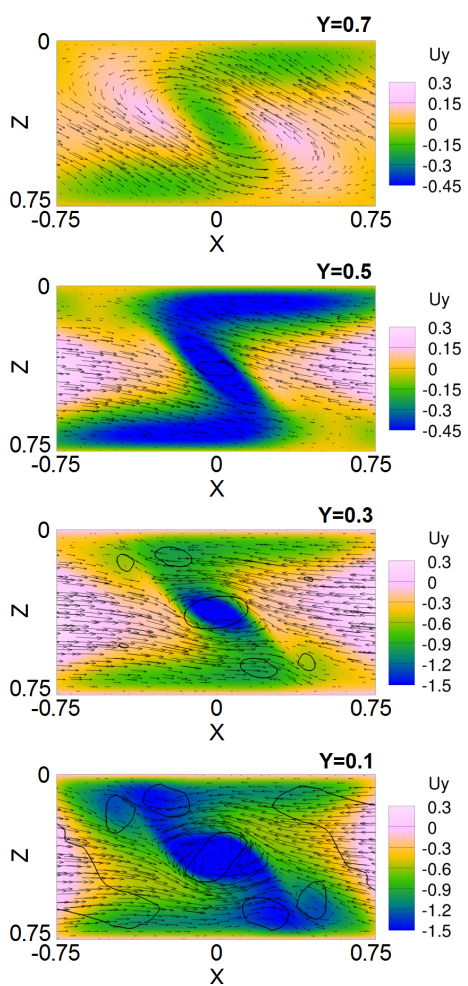

(b)

Figure 16. Flow features in the top part of the arrow-mixer with $\alpha=20^{\circ}$ at $R e=190$ : (a) Isosurface of the $\lambda_{2}$ vortex indicator and velocity streamlines (top left), contours of the in-plane velocity magnitude, velocity streamlines and trace of the isosurface of the $\lambda_{2}$ (thick line) for the sections at $Z=0.375$ (top right), $Z=0.4875$ (bottom left) and $Z=0.6$ (bottom right); (b) contours of the $y$-velocity, in-plane velocity vectors and trace of the isosurface of the $\lambda_{2}$ (thick line) for the cross-sections at (from top to bottom) $Y=0.7, Y=0.5, Y=0.3$ and $Y=0.1$. 


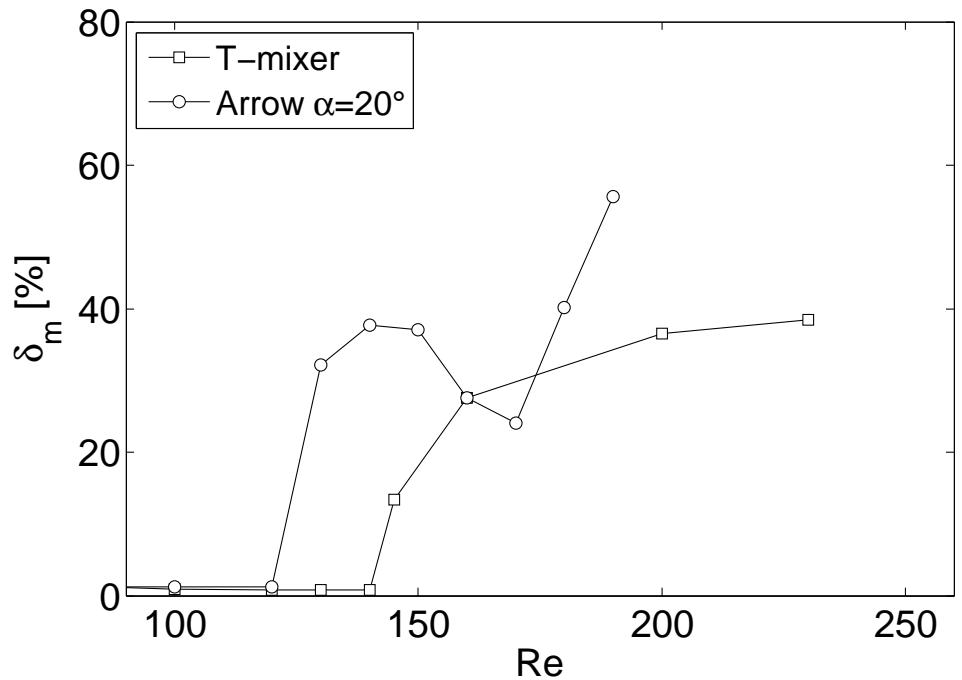

Figure 17. Mixing degree as a function of the Reynolds number for the T-shaped micro-mixer and for the arrow-mixer with $\alpha=20^{\circ}$.

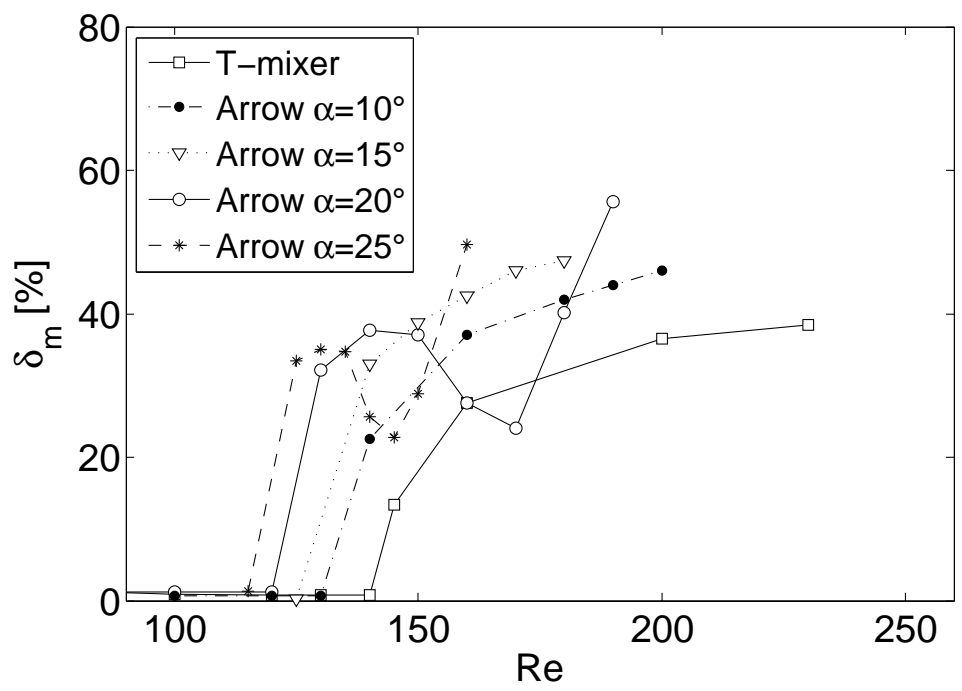

Figure 18. Mixing degree as a function of the Reynolds number for the T-shaped micro-mixer and for the arrow-mixers with different angles. 


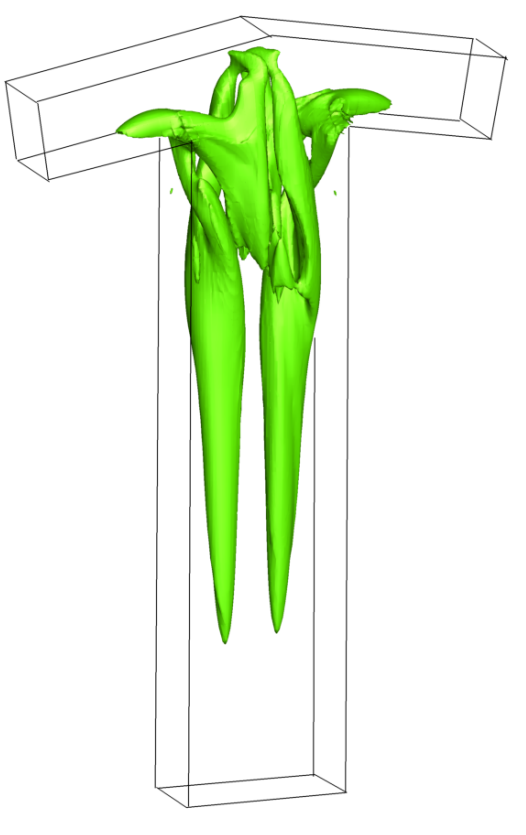

(a)

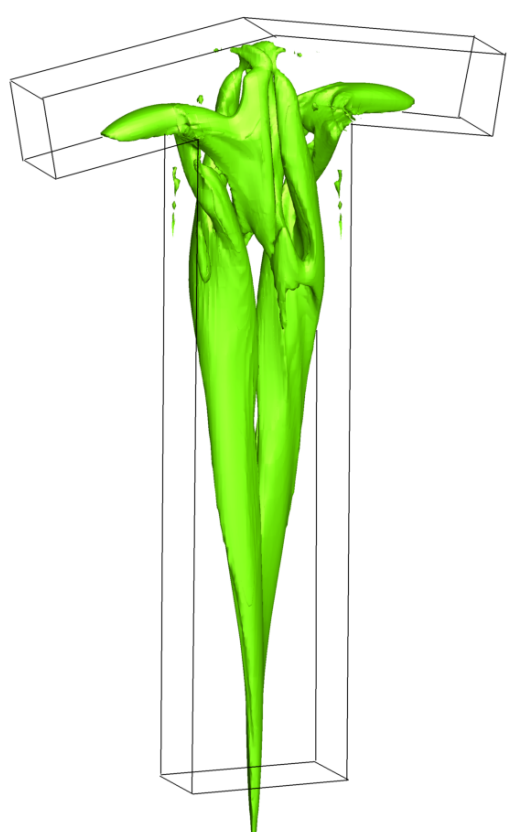

(b)

Figure 19. Isosurface of the $\lambda_{2}$ vortex indicator for the arrow-mixer with $\alpha=10^{\circ}$. Simulations carried out at: (a) $R e=180$ and (b) $R e=200$. 


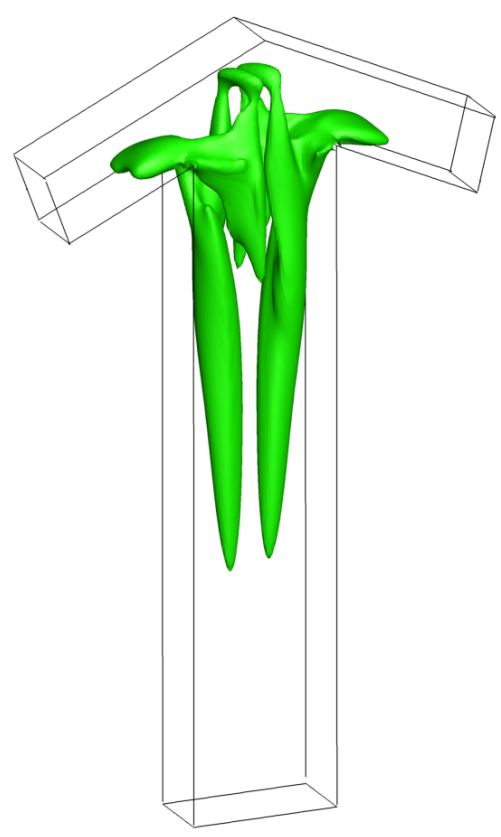

(a)

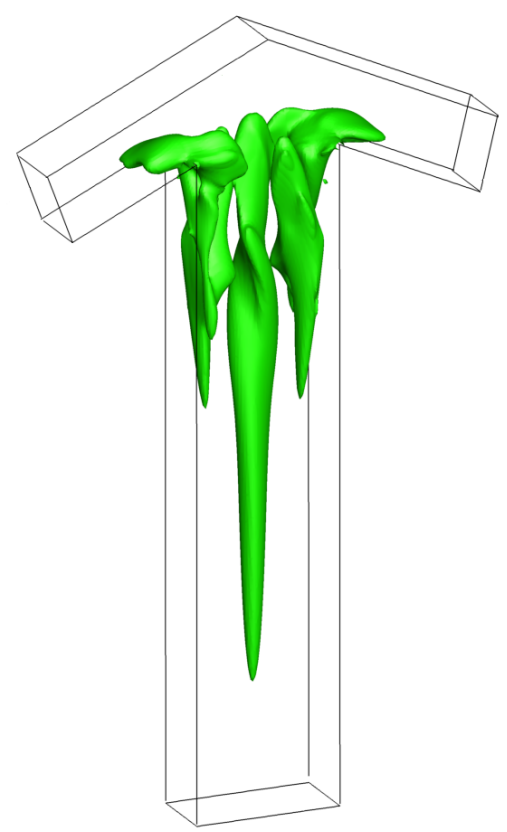

(c)

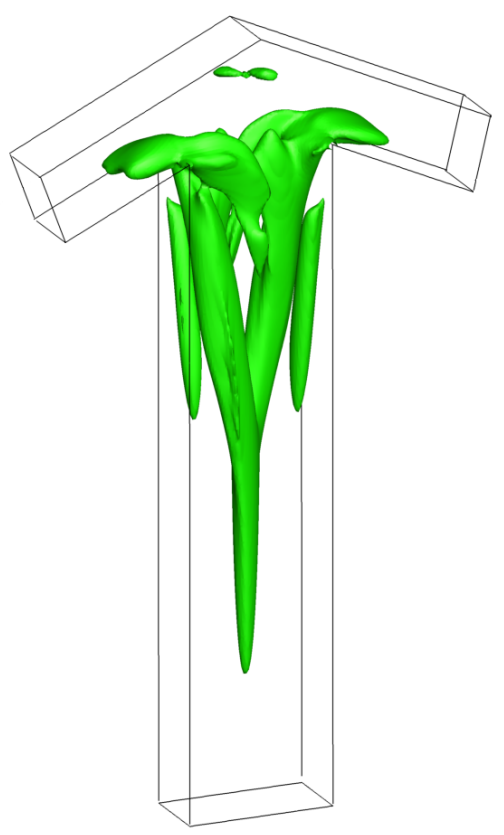

(b)

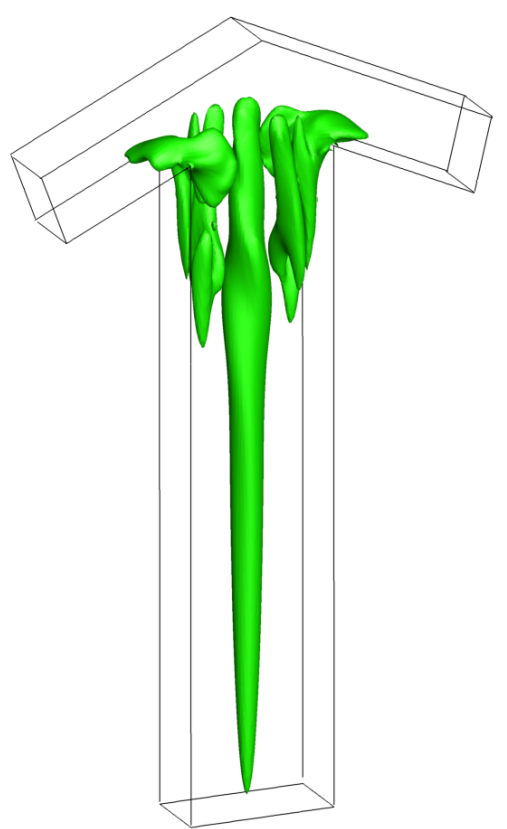

(d)

Figure 20. Isosurface of the $\lambda_{2}$ vortex indicator for the arrow-mixer with $\alpha=25^{\circ}$. Simulations carried out at: (a) $R e=130$, (b) $R e=140$, (c) $R e=150$ and (d) $R e=160$. 